\title{
SPECTRAL SYNTHESIS IN THE MULTIPLIER ALGEBRA OF A $C_{0}(X)$-ALGEBRA
}

\author{
ROBERT J. ARCHBOLD AND DOUGLAS W.B. SOMERSET
}

\begin{abstract}
Let $A$ be a $C_{0}(X)$-algebra with continuous map $\phi$ from $\operatorname{Prim}(A)$, the primitive ideal space of $A$, to a locally compact Hausdorff space $X$. Then the multiplier algebra $M(A)$ is a $C(\beta X)$-algebra with continuous map $\bar{\phi}: \operatorname{Prim}(M(A)) \rightarrow \beta X$ extending $\phi$. For $x \in \operatorname{Im}(\phi)$, let $J_{x}=\bigcap\{P \in \operatorname{Prim}(A): \phi(P)=x\}$ and $H_{x}=\bigcap\{Q \in \operatorname{Prim}(M(A)): \bar{\phi}(Q)=$ $x$ \}. Then $J_{x} \subseteq H_{x} \subseteq \tilde{J}_{x}$, the strict closure of $J_{x}$ in $M(A)$. Thus $H_{x}$ is strictly closed if and only if $H_{x}=\tilde{J}_{x}$, and the 'spectral synthesis' question asks when this happens. In this paper it is shown that, for $\sigma$-unital $A, H_{x}$ is strictly closed for all $x \in \operatorname{Im}(\phi)$ if and only if $J_{x}$ is locally modular for all $x \in \operatorname{Im}(\phi)$ and $\phi$ is a closed map relative to its image. Various related results are obtained.
\end{abstract}

2000 Mathematics Subject Classification: 46L05, 46L57.

\section{INTRODUCTION}

Let $A$ be a $\mathrm{C}^{*}$-algebra with multiplier algebra $M(A)[2,9]$. The ideal structure of $M(A)$ is typically much more complicated than that of $A$ and has been widely studied by a number of authors $[1,16,23,26,27,31,32]$. In $[6,7]$ we investigated certain aspects of the ideal structure of $\mathrm{M}(\mathrm{A})$ which arise from a $C_{0}(X)$-algebra structure on the algebra $A$. The notion of a $C_{0}(X)$-algebra was introduced by Kasparov [22] following extensive earlier work on continuous and upper semi-continuous fields (see, for example, [10, 17, 19, 25, 34]). As noted in [7, Section 1], every $C^{*}$-algebra is a $C_{0}(X)$-algebra, typically in many ways. $C_{0}(X)$ algebras have been studied in $[3,8,13,14,15,20,28]$. In [6, Section 1], we saw that if $A$ is a $C_{0}(X)$-algebra then corresponding to each $x \in X$ there are two natural ideals $H_{x}$ and $\tilde{J}_{x}$ in $M(A)$ which one might hope would be equal but in fact need not be so. The aim of this paper is to characterize the equality $H_{x}=\tilde{J}_{x}$ in terms of $A$ and $X$ (without reference to $M(A))$.

Recall that $A$ is a $C_{0}(X)$-algebra if there is a continuous map $\phi$, called the base map, from $\operatorname{Prim}(A)$, the primitive ideal space of $A$ with the hull-kernel topology, to the locally compact Hausdorff space $X$ [35, Proposition C.5]. We will use $X_{\phi}$ to denote the image of $\phi$ in $X$. If $A$ is a $C_{0}(X)$-algebra then $M(A)$ is a $C(\beta X)$-algebra with continuous map $\bar{\phi}: \operatorname{Prim}(M(A)) \rightarrow \beta X$ (the Stone-Čech compactification of $X$ ) extending $\phi$ (see Section 2).

For $x \in X_{\phi}$, let $J_{x}$ be the closed ideal of $A$ defined by $J_{x}=\bigcap\{P \in \operatorname{Prim}(A): \phi(P)=x\}$ and let $H_{x}$ be the closed ideal of $M(A)$ defined by $H_{x}=\bigcap\{Q \in \operatorname{Prim}(M(A)): \bar{\phi}(Q)=x\}$. Let $\tilde{J}_{x}$ be the strict closure of $J_{x}$ in $M(A)$. Then $J_{x} \subseteq H_{x} \subseteq \tilde{J}_{x}$ and hence $H_{x}$ is strictly closed if and only if $H_{x}=\tilde{J}_{x}$ (see Proposition 2.3). The 'spectral synthesis' question asks for conditions on $A$ and $X$ characterizing when $H_{x}$ is strictly closed. It was shown in [7], for instance, that if $A$ is stable and $\sigma$-unital then for $x \in X_{\phi}, H_{x}$ is strictly closed if and only if $x$ is a $\mathrm{P}$-point in $X_{\phi}$. 
In this paper we return to the question in a more general context. The main result (Corollary 3.7) is that if $A$ is a $\sigma$-unital $C_{0}(X)$-algebra with base map $\phi$ then $H_{x}$ is strictly closed for all $x \in X_{\phi}$ if and only if $J_{x}$ is locally modular for all $x \in X_{\phi}$ and $\phi$ is a closed map relative to its image. Here $J_{x}$ is said to be locally modular if whenever $Q$ lies in the boundary in $\operatorname{Prim}(A)$ of $H(x)=\left\{P \in \operatorname{Prim}(A): P \supseteq J_{x}\right\}$ then there exists a neighbourhood $V$ of $Q$ in $\operatorname{Prim}(A) \backslash U(x)$ (where $U(x)$ is the interior of $H(x)$ ) such that $A / \operatorname{ker} V$ is a unital $C^{*}$-algebra.

If $A$ is separable then the same characterization is valid for spectral synthesis at a point $x \in X_{\phi}$ (Corollary 3.10), namely $H_{x}$ is strictly closed if and only if $J_{x}$ is locally modular and $\phi$ is a locally closed map at $x$. For general $\sigma$-unital $C_{0}(X)$-algebras this condition is close to characterizing spectral synthesis at a point (see Theorem 3.6) but does not quite succeed (Example 4.5), and we have to leave the problem open.

The structure of the paper is as follows. Section 2 gives basic information on $C_{0}(X)$ algebras and spectral synthesis. Section 3 contains some of the main results of the paper, described above. Section 4 looks more closely at pointwise spectral synthesis for a $\sigma$-unital $C_{0}(X)$-algebra $A$ and identifies the points in $X_{\phi}$ which are difficult to deal with. The main result is the characterization of pointwise spectral synthesis in the case when $A$ is a continuous $C_{0}(X)$-algebra (Theorem 4.6).

In Sections 5 and 6 , we restrict to the important special case when $\phi$ is the complete regularization map for $\operatorname{Prim}(A)$ and the connecting order $\operatorname{Orc}(A)$ is finite. In $\operatorname{Section} 5$, we show that, in this case, if $A$ is $\sigma$-unital then the local modularity of $J_{x}$ implies that the complete regularization map $\phi$ is locally closed at $x$. Hence if $A$ is also separable then $H_{x}$ is strictly closed if and only if $J_{x}$ is locally modular (Corollary 5.4). In Section 6, we show that if $J_{x}$ is locally modular then either $J_{x}$ does not contain the centre of $A$ or the hull $H(x)$ of $J_{x}$ in $\operatorname{Prim}(A)$ must have non-empty interior - an unusual property unless $H(x)$ is a clopen set (Corollary 6.3).

Note added in revision.

After this paper was submitted for publication, we learned that David McConnell (private communication) had independently obtained Proposition 2.6(iii), Proposition 2.6(i) (in the case where $\phi$ is the complete regularization map), and versions of Corollary 4.3, Corollary 4.4 and Theorem 4.6 with somewhat stronger hypotheses.

We are grateful to the referee for a number of helpful comments and for pointing out an error in the original proof of Theorem 3.6.

\section{General $C_{0}(X)$-algebras}

In this section we gather some information about $C_{0}(X)$-algebras and establish the basic facts about spectral synthesis (Proposition 2.6). For $C_{0}(X)$-algebras we follow the terminology of $[6]$.

Let $A$ be a $C_{0}(X)$-algebra with base map $\phi: \operatorname{Prim}(A) \rightarrow X$, and recall that $X_{\phi}=\operatorname{Im}(\phi)$. Then $X_{\phi}$ is completely regular; and if $A$ is $\sigma$-unital, $X_{\phi}$ is $\sigma$-compact and hence normal [7, Section 2]. For $x \in X_{\phi}$, set $J_{x}=\bigcap\{P \in \operatorname{Prim}(A): \phi(P)=x\}$, and for $x \in X \backslash X_{\phi}$, set $J_{x}=A$. For $a \in A$, the function $x \rightarrow\left\|a+J_{x}\right\|(x \in X)$ is upper semi-continuous [35, Proposition C.10]. The $C_{0}(X)$-algebra $A$ is said to be continuous if, for all $a \in A$, the norm 
function $x \rightarrow\left\|a+J_{x}\right\|(x \in X)$ is continuous. By Lee's theorem [35, Proposition C.10 and Theorem C.26], this happens if and only if the base map $\phi$ is open.

Let $J$ be a proper, closed, two-sided ideal of a $C^{*}$-algebra $A$. The quotient map $q_{J}: A \rightarrow$ $A / J$ has a canonical extension $\tilde{q_{J}}: M(A) \rightarrow M(A / J)$. We define a proper, closed, two-sided ideal $\tilde{J}$ of $M(A)$ by

$$
\tilde{J}=\operatorname{ker} \tilde{q_{J}}=\{b \in M(A): b a, a b \in J \text { for all } a \in A\} .
$$

The following proposition was proved in [6, Proposition 1.1].

Proposition 2.1. Let $J$ be a proper, closed, two-sided ideal of a $C^{*}$-algebra $A$. Then

(i) $\tilde{J}$ is the strict closure of $J$ in $M(A)$;

(ii) $\tilde{J} \cap A=J$;

(iii) if $P \in \operatorname{Prim}(A)$ then $\tilde{P}$ is primitive (and hence is the unique ideal in $\operatorname{Prim}(M(A)$ ) whose intersection with $A$ is $P)$;

(iv) $\tilde{J}=\bigcap\{\tilde{P}: P \in \operatorname{Prim}(A)$ and $P \supseteq J\}$ and for all $b \in M(A)$

$$
\|b+\tilde{J}\|=\sup \{\|b+\tilde{P}\|: P \in \operatorname{Prim}(A) \text { and } P \supseteq J\}
$$

(v) $(A+\tilde{J}) / \tilde{J}$ is an essential ideal in $M(A) / \tilde{J}$.

Furthermore, the map $P \mapsto \tilde{P}(P \in \operatorname{Prim}(A))$ maps $\operatorname{Prim}(A)$ homeomorphically onto a dense, open subset of $\operatorname{Prim}(M(A))[29,4.1 .10]$. For $S \subseteq \operatorname{Prim}(A)$, we write $\tilde{S}=\{\tilde{P}: P \in S\}$. In view of Proposition 2.1(ii), $(A+\tilde{J}) / \tilde{J}$ is canonically isomorphic to $A / J$. If $A / J$ is unital then $(A+\tilde{J}) / \tilde{J}$ is a unital essential ideal of $M(A) / \tilde{J}$ and therefore equal to $M(A) / \tilde{J}$.

Now suppose that $A$ is a $C_{0}(X)$-algebra, $x \in X$ and $a \in A$. If $A / J_{x}$ is unital, the spectrum of $a+J_{x}$ (in $A / J_{x}$ ) coincides with the spectrum of $a+\tilde{J}_{x}$ in $M(A) / \tilde{J}_{x}$ by the previous remark. If $A / J_{x}$ is non-unital, the spectrum of $a+J_{x}$ (in the unitization of $A / J_{x}$ ) is equal to the spectrum of $a+\tilde{J}_{x}$ in $\left(A+\tilde{J}_{x}\right) / \tilde{J}_{x}+\mathbb{C}\left(1+\tilde{J}_{x}\right)$ and hence in $M(A) / \tilde{J}_{x}[12,1.3 .10(\mathrm{ii})]$.

The following proposition was proved in [6, Proposition 1.2].

Proposition 2.2. Let $A$ be a $C_{0}(X)$-algebra with base map $\phi$. Then $\phi$ has a unique extension to a continuous map $\bar{\phi}: \operatorname{Prim}(M(A)) \rightarrow \beta X$ such that $\bar{\phi}(\tilde{P})=\phi(P)$ for all $P \in \operatorname{Prim}(A)$. Hence $M(A)$ is a $C(\beta X)$-algebra with base map $\bar{\phi}$ and $\operatorname{Im}(\bar{\phi})=\operatorname{cl}_{\beta X}\left(X_{\phi}\right)$.

Now let $A$ be a $C_{0}(X)$-algebra with base map $\phi$ and let $\bar{\phi}: \operatorname{Prim}(M(A)) \rightarrow \beta X$ be as in Proposition 2.2. For $x \in \beta X$, we define $H_{x}=\bigcap\{Q \in \operatorname{Prim}(M(A)): \bar{\phi}(Q)=x\}$, a closed two-sided ideal of $M(A)$. Thus $H_{x}$ is defined in relation to $(M(A), \beta X, \bar{\phi})$ in the same way that $J_{x}$ (for $x \in X$ ) is defined in relation to $(A, X, \phi)$. It follows that for each $b \in M(A)$, the function $x \rightarrow\left\|b+H_{x}\right\|(x \in \beta X)$ is upper semi-continuous.

The next proposition was proved in [7, Proposition 1.3].

Proposition 2.3. Let $A$ be a $C_{0}(X)$-algebra with base map $\phi$, and set $X_{\phi}=\operatorname{Im}(\phi)$.

(i) For all $x \in X, J_{x} \subseteq H_{x} \subseteq \tilde{J}_{x}$ and $J_{x}=H_{x} \cap A$.

(ii) For all $x \in X, H_{x}$ is strictly closed if and only if $H_{x}=\tilde{J}_{x}$.

(iii) For all $b \in M(A),\|b\|=\sup \left\{\left\|b+\tilde{J}_{x}\right\|: x \in X_{\phi}\right\}=\sup \left\{\left\|b+H_{x}\right\|: x \in X_{\phi}\right\}$.

We now turn to the subject of spectral synthesis and our first proposition seeks to justify the use of the name. Recall that in the the theory of commutative Banach algebras, spectral 
synthesis holds at a point $x$ in the maximal ideal space provided that each element of the algebra whose Gelfand transform vanishes at $x$ can be approximated in (the original) norm by elements whose transforms vanish in a neighbourhood of $x$. At this stage we need some more notation.

For a $\mathrm{C}^{*}$-algebra $A$, let $Z(A)$ denote the centre of $A$. Now let $A$ be a $C_{0}(X)$-algebra with base map $\phi$. For $b \in M(A)$, let $Z(b)=\left\{x \in X_{\phi}: b \in \tilde{J}_{x}\right\}$ and let Int $Z(b)$ be the interior of $Z(b)$ relative to $X_{\phi}$. Recall that the Dauns-Hofmann isomorphism $\theta_{A}: C^{b}(\operatorname{Prim}(A)) \rightarrow$ $Z(M(A))$ has the property that $\theta_{A}(f) a+P=f(P)(a+P)$ for $f \in C^{b}(\operatorname{Prim}(A)), a \in A$, and $P \in \operatorname{Prim}(A)$ (equivalently, $\theta_{A}(f)+\tilde{P}=f(P)(1+\tilde{P})$ ).

Proposition 2.4. Let $A$ be a $C_{0}(X)$-algebra with base map $\phi$ and let $x \in X_{\phi}$. Set $H_{x}^{\text {alg }}=$ $\{b \in M(A): x \in \operatorname{Int} Z(b)\}$. Then $H_{x}^{\text {alg }} \subseteq H_{x}$ and $H_{x}$ is the norm-closure of $H_{x}^{\text {alg }}$.

Proof. Let $b \in H_{x}^{\text {alg }}$. Then $x$ lies in the interior $U$ of $Z(b)$ in $X_{\phi}$. There exists $f \in C^{b}\left(X_{\phi}\right)$ such that $f(x)=0$ and $f\left(X_{\phi} \backslash U\right)=\{1\}$. Let $z=\theta_{A}(f \circ \phi) \in Z(M(A))$. Suppose that $Q \in \operatorname{Prim}(M(A))$ and $Q \supseteq H_{x}$. Let $\left(P_{\alpha}\right)$ be a net in $\operatorname{Prim}(A)$ such that $\tilde{P}_{\alpha} \rightarrow Q$. Since $z$ is central,

$$
\|z+Q\|=\lim \left\|z+\tilde{P}_{\alpha}\right\|=\lim \left|f\left(\phi\left(P_{\alpha}\right)\right)\right|=|f(\bar{\phi}(Q))|=|f(x)|=0 .
$$

Thus $z \in Q$ and hence $z \in H_{x}$. For $P \in \operatorname{Prim}(A)$,

$$
z b+\tilde{P}=f(\phi(P))(b+\tilde{P})=b+\tilde{P}
$$

because $b \in \tilde{J}_{\phi(P)} \subseteq \tilde{P}$ whenever $f(\phi(P)) \neq 1$. Hence $z b=b$ and therefore $b \in H_{x}$.

For the second part of the proof, let $b \in H_{x}$ and $\epsilon>0$. By upper semi-continuity, there is an open neighbourhood $U$ of $x$ in $X$ such that $\left\|b+H_{y}\right\|<\epsilon$ for all $y \in U$. Let $V=U \cap X_{\phi}$. There exists a continuous function $f: X_{\phi} \rightarrow[0,1]$ such that $f(x)=0$ and $f\left(X_{\phi} \backslash V\right)=\{1\}$. Define $g:[0,1] \rightarrow[0,1]$ by $g(t)=0(0 \leq t \leq 1 / 2)$ and $g(t)=2 t-1(1 / 2<t \leq 1)$. Let $h=g \circ f$. Then $h\left(X_{\phi} \backslash V\right)=\{1\}$ and there exists an open neighbourhood $W$ of $x$ in $X_{\phi}$ such that $\left.h\right|_{W}=0$. Let $z=\theta_{A}(h \circ \phi) \in Z(M(A))$.

Suppose that $y \in W, P \in \operatorname{Prim}(A)$ and $\phi(P)=y$. Then $z b+\tilde{P}=h(y)(b+\tilde{P})=0$. It follows that $z b \in \tilde{J}_{y}$ and hence $z b \in H_{x}^{\text {alg }}$.

Finally, for $P \in \operatorname{Prim}(A)$,

$$
\|(b-b z)+\tilde{P}\|=(1-h(\phi(P)))\|b+\tilde{P}\| \leq(1-h(\phi(P)))\left\|b+H_{\phi(P)}\right\|<\epsilon
$$

since $h(\phi(P))=1$ if $\phi(P) \notin U$. Hence $\|b-z b\|<\epsilon$.

It follows from Proposition 2.3 and Proposition 2.4 that, for $x \in X_{\phi}$, the ideal $H_{x}$ is strictly closed if and only if every element $b \in M(A)$ which vanishes at $x$ can be approximated in norm by elements vanishing in a neighbourhood of $x$ in $X_{\phi}$. Thus we think of $H_{x}$ being strictly closed as corresponding to 'spectral synthesis holding at $x$ '.

As in [6], we define $\mu: C_{0}(X) \rightarrow Z(M(A))$ by $\mu(f)=\theta_{A}(f \circ \phi)\left(f \in C_{0}(X)\right)$ Now set $Z^{\prime}(A)=\mu\left(C_{0}(X)\right) \cap A$ and note that $Z^{\prime}(A) \subseteq Z(A)$. The next lemma was proved in $[6$, Lemma 2.1].

Lemma 2.5. Let $A$ be a $C_{0}(X)$-algebra with base map $\phi$ and let $x \in X_{\phi}$. Then $J_{x} \supseteq Z^{\prime}(A)$ if and only if there exists $R \in \operatorname{Prim}(M(A))$ with $R \supseteq A$ such that $\bar{\phi}(R)=x$.

We can now give the basic results on spectral synthesis for general $C_{0}(X)$-algebras. 
Proposition 2.6. Let $A$ be a $C_{0}(X)$-algebra with base map $\phi$ and let $x \in X_{\phi}$.

(i) If $J_{x} \nsupseteq Z^{\prime}(A)$ then $H_{x}$ is strictly closed in $M(A)$.

(ii) If $x$ is an isolated point in $X_{\phi}$ then $H_{x}$ is strictly closed in $M(A)$.

(iii) If $J_{x} \supseteq Z^{\prime}(A)$ and $A / J_{x}$ is unital then $H_{x}$ is not strictly closed in $M(A)$.

Proof. (i) This was proved in [6, Proposition 2.2].

(ii) Since $x$ is an isolated point in $X_{\phi}, W=\phi^{-1}(x)$ is a clopen subset of $\operatorname{Prim}(A)$. Set $Y=\operatorname{Prim}(A) \backslash W$. Suppose that $R \in \operatorname{Prim}(M(A))$ and $R \nsupseteq \tilde{J}_{x}$. Then $R$ is not in the closure of the set $\{\tilde{P}: P \in W\}$. Since the set $\{\tilde{P}: P \in \operatorname{Prim}(A)\}$ is dense in $\operatorname{Prim}(M(A))$, $R$ must lie in the closure of the set $\{\tilde{P}: P \in Y\}$. Hence the continuity of $\bar{\phi}$ implies that $\bar{\phi}(R)$ lies in the closure of $\{\phi(P): P \in Y\}$. Thus $\bar{\phi}(R) \in X \backslash\{x\}$ and so $R \nsupseteq H_{x}$.

(iii) Let $p \in A$ such that $p+J_{x}$ is the identity for $A / J_{x}$. Then $a p-a, p a-a \in J_{x}$ for all $a \in A$ and hence $1-p \in \tilde{J}_{x}$. On the other hand, by Lemma 2.5 there exists $R \in \operatorname{Prim}(M(A))$ with $R \supseteq A$ such that $\bar{\phi}(R)=x$. Since $R \supseteq A, p \in R$, and hence $1-p \notin R$. Thus $1-p \notin H_{x}$.

If $J_{x} \nsupseteq Z^{\prime}(A)$ then $A / J_{x}$ is unital [6, Proposition 2.2]. So the three cases of Proposition 2.6 cover all possibilities for $x$ except when $x$ is a non-isolated point of $X_{\phi}$ with $A / J_{x}$ non-unital. This is the case of interest which will occupy us for the rest of the paper. We will use the following notation. Let $U_{\phi}=\left\{x \in X_{\phi}: J_{x} \nsupseteq Z^{\prime}(A)\right\}$, an open subset of $X_{\phi}$ (see [6, Section $2]$ ); and let $W_{\phi}=X_{\phi} \backslash U_{\phi}$. Let $\partial U_{\phi}$ denote the boundary of $U_{\phi}$ in $X_{\phi}$.

Finally in this section, we consider spectral synthesis for a closed subset $E$ of $X_{\phi}$. Define $J_{E}=\bigcap\{P \in \operatorname{Prim}(A): \phi(P) \in E\}, H_{E}=\bigcap\{Q \in \operatorname{Prim}(M(A)): \bar{\phi}(P) \in E\}$ and $H_{E}^{\text {alg }}=\{b \in M(A): E \subseteq$ Int $Z(b)\}$. In the following analogue of Proposition 2.4, we restrict to the case where $A$ is $\sigma$-unital in order to ensure that $X_{\phi}$ is normal.

Proposition 2.7. Let $A$ be a $\sigma$-unital $C_{0}(X)$-algebra with base map $\phi$ and let $E$ be a closed subset of $X_{\phi}$. Then $H_{E}^{\text {alg }} \subseteq H_{E}$ and $H_{E}$ is the norm-closure of $H_{x}^{\text {alg }}$.

Proof. Let $b \in H_{E}^{\text {alg }}$. Then $E$ is contained in the interior $U$ of $Z(b)$ in $X_{\phi}$. Since $X_{\phi}$ is normal, there exists $f \in C^{b}\left(X_{\phi}\right)$ such that $f(E)=\{0\}$ and $f\left(X_{\phi} \backslash U\right)=\{1\}$. Let $z=\theta_{A}(f \circ \phi) \in Z(M(A))$. As in the proof of Proposition 2.4, $z \in H_{E}$ and $b=z b \in H_{E}$.

For the second part of the proof, let $b \in H_{E}$ and $\epsilon>0$. By upper semi-continuity, there is an open neighbourhood $U$ of $E$ in $X$ such that $\left\|b+H_{y}\right\|<\epsilon$ for all $y \in U$. Let $V=U \cap X_{\phi}$. Since $X_{\phi}$ is normal, there exists an open neighbourhood $W$ of $E$ in $X_{\phi}$, with closure contained in $V$, and a continuous function $f: X_{\phi} \rightarrow[0,1]$ such that $f(W)=\{0\}$ and $f\left(X_{\phi} \backslash V\right)=\{1\}$. Let $z=\theta_{A}(f \circ \phi) \in Z(M(A))$. As in the proof of Proposition 2.4, $z b \in H_{x}^{\text {alg }}$ and $\|b-z b\|<\epsilon$.

Let $A$ be a $\sigma$-unital $C_{0}(X)$-algebra with base map $\phi$ and let $E$ be a closed subset of $X_{\phi}$. If $P \in \operatorname{Prim}(A)$ and $P \supseteq J_{E}$ then $\phi(P) \in E$ because $\phi$ is continuous and $E$ is closed. Hence

$$
J_{E}=\bigcap_{x \in E} J_{x} \subseteq \bigcap_{x \in E} H_{x}=H_{E} \subseteq \bigcap_{x \in E} \tilde{J}_{x}=\tilde{J}_{E},
$$

by Proposition 2.1(iv). It follows from Proposition 2.1(i) and Proposition 2.7 that $H_{E}^{\text {alg }}$ is dense in $\bigcap_{x \in E} \tilde{J}_{x}$ (which may be thought of as spectral synthesis for E) if and only if $H_{E}$ is strictly closed in $M(A)$. We therefore define spectral synthesis for $E$ to mean that $H_{E}$ is strictly closed in $M(A)$. If $E$ and $F$ are closed subsets of $X_{\phi}$ then $H_{E \cup F}=H_{E} \cap H_{F}$. 
In contrast to the theory of commutative Banach algebras, it follows that if $E$ and $F$ have spectral synthesis then so does $E \cup F$.

We shall see in Proposition 2.9 that the question of spectral synthesis for a closed subset $E$ of $X_{\phi}$ (i.e. whether $H_{E}$ is strictly closed in $M(A)$ ) can be reduced to the question of spectral synthesis for a singleton, but at the expense of changing the base map $\phi$. We will need the following standard topological lemma, where $X / E$ is the quotient space of a topological space $X$ obtained by identifying all of the points in a given subset $E$.

Lemma 2.8. Let $X$ be a normal, Hausdorff space and let $E$ be a non-empty closed subset. Set $Y=X / E$. Then $Y$ is normal and Hausdorff and hence completely regular.

Proof. Let $q: X \rightarrow Y$ be the quotient map. For $y \in Y, q^{-1}(y)$ is closed and so $Y$ is a $T_{1}$ space. Let $B$ and $C$ be disjoint, non-empty closed subsets of $Y$. Then $G=q^{-1}(B)$ and $H=q^{-1}(C)$ are disjoint closed sets in the normal space $X$, and hence there exist disjoint open sets $U$ and $V$ such that $G \subseteq U$ and $H \subseteq V$. Without loss of generality, we may assume that $G \nsupseteq E$ and hence $G \cap E=\emptyset$. Thus, replacing $U$ by $U \backslash E$, we may assume that $U$ does not meet $E$. Hence $U$ is saturated with respect to the equivalence relation corresponding to $q$ and so $q(U)$ is an open neighbourhood of $B$ and is disjoint from $q(V)$. If $H \supseteq E$ then $V$ is saturated and so $q(V)$ is open, and if $H \nsupseteq E$ then $V \backslash E$ is a saturated open set containing $H$ and so $q(V \backslash E)$ is an open neighbourhood of $C$. Hence $Y$ is normal, and being $T_{1}$, it is also Hausdorff and completely regular.

Note that if $X$ is Hausdorff but non-normal then $X$ has a closed set $E$ such that $X / E$ is not completely regular. To see this, let $E$ and $F$ be disjoint closed subsets of $X$ which cannot be separated by disjoint open sets. Let $Y=X / E$ with quotient map $q$ and set $q(E)=e$. Then $e$ and the closed set $q(F)$ cannot be separated by disjoint open sets, so $Y$ is not even regular.

Now let $A$ be a $C_{0}(X)$-algebra with base map $\phi$. Let $E$ be a non-empty closed subset of $X_{\phi}$. Set $Y=X_{\phi} / E$ and let $q: X_{\phi} \rightarrow Y$ be the quotient map. If $A$ is $\sigma$-unital then $Y$ is completely regular by Lemma 2.8 and so $A$ is a $C(\beta Y)$-algebra with base map $\psi=q \circ \phi$. Let $e=q(E)$. The next proposition relates $J_{E}$ and $J_{e}$, and $H_{E}$ and $H_{e}$.

Note that if $f \in C^{b}(\operatorname{Prim}(A))$ then the element $\theta_{A}(f) \in Z(M(A))$ induces a function $\bar{f} \in C(\operatorname{Prim}(M(A))$ such that

$$
\theta_{A}(f)+Q=\bar{f}(Q)(1+Q) \quad(Q \in \operatorname{Prim}(M(A))) .
$$

In particular, $\bar{f}(\tilde{P})=f(P)$ for all $P \in \operatorname{Prim}(A)$.

Proposition 2.9. Let $A$ be a $\sigma$-unital $C_{0}(X)$-algebra with base map $\phi$ and let $E$ be a nonempty closed subset of $X_{\phi}$. Set $Y=X_{\phi} / E$ and let $q: X_{\phi} \rightarrow Y$ be the quotient map. Let $\psi=q \circ \phi$. Then $A$ is a $C(\beta Y)$-algebra with base map $\psi$, and $J_{e}=J_{E}$ and $H_{e}=H_{E}$, where $\{e\}=q(E)$.

Proof. For $P \in \operatorname{Prim}(A), \phi(P) \in E$ if and only if $\psi(P)=e$ and therefore $J_{E}=J_{e}$.

For $P \in \operatorname{Prim}(A), \overline{q \circ \phi}(\tilde{P})=(q \circ \phi)(P)=\bar{q}(\bar{\phi}(\tilde{P}))$ and hence $\overline{q \circ \phi}=\bar{q} \circ \bar{\phi}$ by continuity. Thus if $Q \in \operatorname{Prim}(M(A))$ and $\bar{\phi}(Q) \in E$ then $\bar{\psi}(Q)=e$. Hence $H_{E} \supseteq H_{e}$. For the reverse inclusion, suppose that $Q \in \operatorname{Prim}(M(A))$ with $Q \notin W:=\left\{R \in \operatorname{Prim}(M(A)): R \supseteq H_{E}\right\}$. Let $D$ be a compact neighbourhood of $Q$ in $\operatorname{Prim}(M(A))$ with $D$ disjoint from $W$. Then 
$F:=\bar{\phi}(D)$ is a compact subset of $\beta X_{\phi}$ and hence $L:=F \cap X_{\phi}$ is closed in $X_{\phi}$ and disjoint from $E$, and $\bar{\phi}(Q)$ lies in the closure of $L$ in $\beta X_{\phi}$. Since $X_{\phi}$ is normal, $\bar{\phi}(Q)$ does not lie in the closure of $E$ in $\beta X_{\phi}$ and hence there is a continuous, real-valued function $f$ on $\beta X_{\phi}$ such that $f(\bar{\phi}(Q))=1$ and $f(E)=\{0\}$. There is a well-defined function $g: Y \rightarrow \mathbf{R}$ such that $g \circ q=\left.f\right|_{X_{\phi}}$. Let $U$ be an open subset of $\mathbf{R}$. Then $q^{-1}\left(g^{-1}(U)\right)=f^{-1}(U) \cap X_{\phi}$, an open subset of $X_{\phi}$. Thus $g^{-1}(U)$ is open in $Y$ and so $g$ is continuous. Let $\bar{g}$ be the extension of $g$ to a continuous function on $\beta Y$. Then $\bar{g} \circ \bar{q}=f$ by continuity and so $\bar{g} \circ \bar{q} \circ \bar{\phi}(Q)=1$. Hence $\bar{\psi}(Q)=\bar{q} \circ \bar{\phi}(Q) \neq e$. Thus $Q \nsupseteq H_{e}$, and hence $H_{E}=H_{e}$.

\section{Global And LOCAL SPECTRAL Synthesis}

In this section we characterize 'global spectral synthesis' by showing that, for a $\sigma$-unital $C_{0}(X)$-algebra $A, H_{x}$ is strictly closed for all $x \in X_{\phi}$ if and only if $J_{x}$ is locally modular for all $x \in X_{\phi}$ and $\phi$ is a closed map relative to its image (Corollary 3.7). For separable $A$ we can also characterize 'spectral synthesis at a point': if $A$ is separable then for $x \in X_{\phi}, H_{x}$ is strictly closed if and only if $J_{x}$ is locally modular and $\phi$ is locally closed at $x$ (Corollary 3.10 ).

We begin by analyzing the property of $H_{x}$ being strictly closed into two separate subproperties (Proposition 3.2). For this, we need the following lemma.

Lemma 3.1. Let $A$ be a $\sigma$-unital $C^{*}$-algebra and let $Y$ and $Z$ be disjoint closed subsets of $\operatorname{Prim}(A)$. Then the closures of $\tilde{Y}$ and $\tilde{Z}$ are disjoint in $\operatorname{Prim}(M(A))$.

Proof. Set $J=\operatorname{ker}(Y \cup Z)$ and let $B=A / J$. Let $\pi: A \rightarrow B$ be the quotient map. Then the map $P \mapsto \pi(P)(P \in Y \cup Z)$ carries $Y \cup Z$ homeomorphically onto $\operatorname{Prim}(B)$. Thus there exists $b \in Z(M(B))$ such that $b+\pi(P)^{\sim}=1+\pi(P)^{\sim}$ for $P \in Y$ and $b+\pi(P)^{\sim}=0$ for $P \in Z$. By [30, Theorem 10] the canonical map $M(A) \rightarrow M(B)$ is surjective, and hence there exists $a \in M(A)$ such that $a+\tilde{P}=1+\tilde{P}$ for $P \in Y$ and $a+\tilde{P}=0$ for $P \in Z$. Thus, by definition of the hull-kernel topology, $a+Q=0$ for all $Q$ in the closure of $\tilde{Z}$ in $\operatorname{Prim}(M(A))$, and by considering $(1-a)$ we see that likewise $a+Q=1+Q$ for all $Q$ in the closure of $\tilde{Y}$ in $\operatorname{Prim}(M(A))$. Hence $\tilde{Y}$ and $\tilde{Z}$ have disjoint closures in $\operatorname{Prim}(M(A))$.

The first of the two sub-properties into which we analyze the property of strict closure is as follows. Recall that $H(x)=\phi^{-1}(x)\left(x \in X_{\phi}\right)$. For $x \in X_{\phi}$ we say that the base map $\phi$ is locally closed at $x[24, \S 13$.XIV] if whenever $Y$ is a closed subset of $\operatorname{Prim}(A)$ such that $x$ lies in the closure of $\phi(Y)$ then $x \in \phi(Y)$, that is, $Y \cap H(x)$ is non-empty. For example, if $x$ is an isolated point in $X_{\phi}$ (which implies that $H(x)$ is a clopen subset of $\left.\operatorname{Prim}(A)\right)$ then $\phi$ is trivially locally closed at $x$. Note, however, that $H(x)$ could be clopen, yet $x$ non-isolated in $X_{\phi}$. In this case, $\phi$ would not be locally closed at $x$, see Example 4.8(ii). We say that $\phi$ is relatively closed if $\phi(Y)$ is closed in $X_{\phi}$ for all closed subsets $Y$ of $\operatorname{Prim}(A)$. Clearly $\phi$ is relatively closed if and only if $\phi$ is locally closed at each $x \in X_{\phi}$.

Proposition 3.2. Let $A$ be a $C_{0}(X)$-algebra with base map $\phi$ and let $x \in X_{\phi}$. Consider the following properties:

(i) $H_{x}$ is strictly closed;

(ii) $\phi$ is locally closed at $x$;

(iii) for each $b \in \tilde{J}_{x}$ and $\epsilon>0$ there is an open set $V \subseteq \operatorname{Prim}(A)$ with $H(x) \subseteq V$ such that $\|b+\tilde{P}\|<\epsilon$ for all $P \in V$. 
Then (i) $\Rightarrow($ iii) and (ii) $+($ iii) $\Rightarrow(\mathrm{i})$. If $A$ is $\sigma$-unital then (i) $\Rightarrow($ ii) and hence (i) is equivalent to (ii) + (iii).

Proof. (i) $\Rightarrow$ (iii) Suppose that $H_{x}=\tilde{J}_{x}$ and let $b \in \tilde{J}_{x}$ and $\epsilon>0$. Then by the upper semicontinuity of norm functions there is an neighbourhood $U$ of $x$ in $X_{\phi}$ such that $\left\|b+H_{y}\right\|<\epsilon$ for all $y \in U$. Set $V=\phi^{-1}(U)$. Then $H(x) \subseteq V$ and for all $P \in V$

$$
\|b+\tilde{P}\| \leq\left\|b+\tilde{J}_{\phi(P)}\right\| \leq\left\|b+H_{\phi(P)}\right\|<\epsilon .
$$

(ii) + (iii) $\Rightarrow$ (i) Let $b \in \tilde{J}_{x}$ and let $\epsilon>0$ be given. Then by assumption there is an open set $V \subseteq \operatorname{Prim}(A)$ with $H(x) \subseteq V$ such that $\|b+\tilde{P}\|<\epsilon$ for all $P \in V$. Set $Y=\operatorname{Prim}(A) \backslash V$. Then $Y$ is closed and $Y \cap H(x)$ is empty. Since $\phi$ is locally closed at $x$, it follows that $x$ does not belong to the closure of $\phi(Y)$ in $X_{\phi}$. Hence there exists $g \in C^{b}\left(X_{\phi}\right)$ with $0 \leq g \leq 1$ such that $g(\phi(Y))=\{1\}$ and $g(x)=0$. Let $z=\theta_{A}(g \circ \phi) \in Z(M(A))$ so that $0 \leq z \leq 1$ and $z+\tilde{Q}=g(\phi(Q))(1+\tilde{Q})$ for all $Q \in \operatorname{Prim}(A)$. Let $R \in \operatorname{Prim}(M(A))$ with $\bar{\phi}(R)=x$. There is a net $\left(P_{\alpha}\right)$ in $\operatorname{Prim}(A)$ such that $\tilde{P}_{\alpha} \rightarrow R$. Then $x=\bar{\phi}(R)=\lim \phi\left(P_{\alpha}\right)$ and so $g\left(\phi\left(P_{\alpha}\right)\right) \rightarrow g(x)=0$. Thus $\left\|z+\tilde{P}_{\alpha}\right\| \rightarrow 0$ and so, since $z \in Z(M(A)),\|z+R\|=0$. It follows that $z \in H_{x}$ and hence $z b \in H_{x}$. But

$$
\|z b-b\|=\sup \{\|(z b-b)+\tilde{Q}\|: Q \in \operatorname{Prim}(A)\}=\sup \{\|(z b-b)+\tilde{Q}\|: Q \in V\}<\epsilon .
$$

Since $\epsilon$ was arbitrary, $b \in H_{x}$ and hence $\tilde{J}_{x}=H_{x}$.

(i) $\Rightarrow$ (ii) (assuming that $A$ is $\sigma$-unital). Suppose that $\phi$ is not locally closed at $x$ and let $Y$ be a closed subset of $\operatorname{Prim}(A)$ such that $x$ lies in the closure of $\phi(Y)$ but $H(x) \cap Y$ is empty. Let $W$ be the closure of $\tilde{Y}$ in $\operatorname{Prim}(M(A))$. Then $\bar{\phi}(W)$ is a compact and hence closed subset of $\beta X$ containing $\phi(Y)$. Hence there exists $R \in W$ such that $\bar{\phi}(R)=x$ and therefore $R \supseteq H_{x}$. By Proposition 2.1(iv), the closure of $\tilde{H}(x)=\left\{\tilde{P}: P \in \phi^{-1}(x)\right\}$ in $\operatorname{Prim}(M(A))$ is equal to $\operatorname{Prim}\left(M(A) / \tilde{J}_{x}\right)$ (where the latter is identified with the hull of $\tilde{J}_{x}$ in $\operatorname{Prim}(M(A))$ ). But, since $A$ is $\sigma$-unital, $W$ is disjoint from $\operatorname{Prim}\left(M(A) / \tilde{J}_{x}\right)$ by Lemma 3.1 Thus $R \nsupseteq \tilde{J}_{x}$ and so $\tilde{J}_{x} \neq H_{x}$.

In particular, we notice that if $A$ is $\sigma$-unital then a necessary condition for spectral synthesis at $x \in X_{\phi}$ is that $\phi$ should be locally closed at $x$.

To understand the second sub-property (property (iii) of Proposition 3.2) into which we have analyzed the property of being strictly closed, we introduce the idea of local modularity. To define this it is helpful to have the following notation. For $x \in X_{\phi}$, let $\partial H(x)$ be the boundary and $U(x)$ the interior of $H(x)$ in $\operatorname{Prim}(A)$. We say that $J_{x}$ is locally modular if for each $P \in \partial H(x)$ there exists a relatively open neighbourhood $V$ of $P$ in $\operatorname{Prim}(A) \backslash U(x)$ such that $A / \operatorname{ker} V$ is a unital $\mathrm{C}^{*}$-algebra.

For instance, if $x$ is an isolated point of $X_{\phi}$ then $H(x)$ is clopen in $\operatorname{Prim}(A)$ and so $\partial H(x)$ is empty and hence $J_{x}$ is vacuously locally modular. Secondly, if $J_{x} \nsupseteq Z^{\prime}(A)$ (that is, $x \in U_{\phi}$ ) then by upper semi-continuity of norm functions and functional calculus we may find $z \in Z^{\prime}(A)$ such that $z+J_{y}=1_{A / J_{y}}$ for all $y$ in an open neighbourhood $V$ of $x$ in $X_{\phi}$ (cf. the proof of [6, Proposition 2.3]). Hence $z+P=1_{A / P}$ for all $P \in W=\phi^{-1}(V)$, and $H(x) \subseteq W$, so again $J_{x}$ is locally modular. On the other hand, if there exists $P \in \partial H(x)$ such that $A / P$ is non-unital then clearly $J_{x}$ is not locally modular. 
The definition of local modularity is intrinsic to $A$, in that it does not mention $M(A)$, and it is a condition that should be possible to check in concrete cases. The following equivalent condition, however, seems easier to work with, although it does involve $M(A)$. To describe this, we need a slight variant of the definition of $\sim$. Recall from [33] that for $P, Q \in \operatorname{Prim}(A)$ we write $P \sim Q$ if $P$ and $Q$ cannot be separated by disjoint open subsets of $\operatorname{Prim}(A)$ (for a fuller discussion, see Section 5 below). For the multiplier algebra $M(A)$ of a $C_{0}(X)$-algebra $A$, we define $\sim_{x}$ as follows. For $x \in X_{\phi}$ and $Q, R \in \operatorname{Prim}(M(A)) \backslash \tilde{U}(x)$ we say that $Q \sim_{x} R$ if there is a net $\left(P_{\alpha}\right)$ in $\operatorname{Prim}(A) \backslash U(x)$ such that $\left(\tilde{P}_{\alpha}\right)$ converges to both $Q$ and $R$. If $H(x)$ has empty interior then $\sim_{x}$ coincides with the relation $\sim$ on $\operatorname{Prim}(M(A))$ because the canonical image of $\operatorname{Prim}(A)$ is dense in $\operatorname{Prim}(M(A))$. Otherwise $Q \sim_{x} R \Rightarrow Q \sim R$, but the converse need not hold.

For the next lemma, we need the definition of a primal ideal and of the topology $\tau_{s}$. An ideal $J$ in a $\mathrm{C}^{*}$-algebra $A$ is primal if whenever $I_{1}, \ldots, I_{n}$ is a finite collection of ideals of $A$ with the product $I_{1} \ldots I_{n}=\{0\}$ then $I_{i} \subseteq J$ for at least one $i \in\{1, \ldots, n\}$ [5]. Every primitive ideal is prime and hence primal. The set of proper primal ideals of $A$ is denoted $\operatorname{Primal}^{\prime}(A)$. The $\tau_{s}$ topology on $\operatorname{Primal}^{\prime}(A)$ is defined to be the weakest topology for which all the norm functions $I \rightarrow\|a+I\|\left(a \in A, I \in \operatorname{Primal}^{\prime}(A)\right)$ are continuous (see [17, Section II]). If $A$ is unital then $\operatorname{Primal}^{\prime}(A)$ is $\tau_{s}$-compact [4, Proposition 4.1].

Lemma 3.3. Let $A$ be a $C_{0}(X)$-algebra with base map $\phi$ and let $x \in X_{\phi}$. Consider the following conditions:

(i) $J_{x}$ is locally modular;

(ii) for all $P \in \partial H(x)$ and $R \in \operatorname{Prim}(M(A) / A), \tilde{P} \chi_{x} R$.

Then (i) $\Rightarrow($ ii), and (i) and (ii) are equivalent if $A$ is $\sigma$-unital.

Proof. Suppose first that (i) holds, and let $P \in \partial H(x)$. Let $V$ be an open neighbourhood of $P$ in $\operatorname{Prim}(A) \backslash U(x)$ such that $A / \operatorname{ker} V$ is unital. Let $W$ be the closure of $V$ in $\operatorname{Prim}(A)$, so that $\operatorname{ker} V=\operatorname{ker} W$. Write $J=\operatorname{ker} W($ so that $W=\operatorname{Prim}(A / J))$ and recall that the quotient map $q_{J}: A \rightarrow A / J$ has a canonical extension $\tilde{q}_{J}: M(A) \rightarrow M(A / J)=A / J$. For each $b \in M(A)$ there exists $a \in A$ such that $b-a \in \operatorname{ker} \tilde{q}_{J}=\tilde{J}$, so $A+\tilde{J}=M(A)$. Thus if $R \in \operatorname{Prim}(M(A) / A)$ then $R \nsupseteq \tilde{J}$. It follows that $\tilde{W}=\operatorname{Prim}(M(A) / \tilde{J})$, a closed subset of $\operatorname{Prim}(M(A))$. Hence if $\left(P_{\alpha}\right)$ is a net in $\operatorname{Prim}(A) \backslash U(x)$ with $P_{\alpha} \rightarrow P$ then eventually $P_{\alpha} \in V$, so all the limits of the net $\left(\tilde{P}_{\alpha}\right)$ in $\operatorname{Prim}(M(A))$ lie in $\tilde{W}$. Thus $\tilde{P} \chi_{x} R$ for any $R \in \operatorname{Prim}(M(A) / A)$.

Conversely, suppose that (ii) holds and that $A$ is $\sigma$-unital. Let $u$ be a strictly positive element in $A$ with $\|u\|=1$. Let $Q \in \partial H(x)$ and suppose that $A+\tilde{Q} \neq M(A)$. Then there exists a maximal ideal $M$ of $M(A)$ with $M \supseteq A+\tilde{Q}$, and hence $M \sim_{x} \tilde{Q}$ contradicting (ii). Thus $A+\tilde{Q}=M(A)$, so $A / Q$ is unital and $\|(1-u)+\tilde{Q}\|<1$.

Let $P \in \partial H(x)$ and suppose, for a contradiction, that there is a net $\left(P_{\alpha}\right)$ in $\operatorname{Prim}(A) \backslash U(x)$ with $P_{\alpha} \rightarrow P$ and $\left\|(1-u)+\tilde{P}_{\alpha}\right\| \rightarrow 1$. By the $\tau_{s}$-compactness of $\operatorname{Primal}^{\prime}(M(A))$, and by passing to a subnet if necessary, we may assume that there exists $J \in \operatorname{Primal}^{\prime}(M(A))$ such that $\tilde{P}_{\alpha} \rightarrow J\left(\tau_{s}\right)$. Hence $\|(1-u)+J\|=1$ and so there exists $R \in \operatorname{Prim}(M(A) / J)$ such that $\|(1-u)+R\|=1[12,3.3 .6]$. Since $\tilde{P}_{\alpha} \rightarrow J\left(\tau_{s}\right), \tilde{P}_{\alpha} \rightarrow R$ and so $R \nsupseteq A$ by (ii). Then $R=\tilde{Q}$, where $Q:=R \cap A \in \operatorname{Prim}(A)$, and so $P_{\alpha} \rightarrow Q$. Since $\phi$ is continuous, $\phi(Q)=\phi(P)=x$ and so $Q \in H(x)$. But $P_{\alpha} \in \operatorname{Prim}(A) \backslash U(x)$ for all $\alpha$ and so $Q \in \partial H(x)$, contradicting the 
fact that $\|(1-u)+\tilde{Q}\|=1$. Thus no such net $\left(P_{\alpha}\right)$ exists and so there exists $\epsilon>0$ and a neighbourhood $V$ of $P$ in $\operatorname{Prim}(A) \backslash U(x)$ such that $\|(1-u)+\tilde{Q}\|<1-\epsilon$ for all $Q \in V$.

Now let $f$ be a continuous function on $[0,1]$ with $f(0)=0, f([\epsilon, 1])=\{1\}$, and $\|f\|=1$, and set $v=f(u)$. Then $(1-v)+\tilde{Q}=0$ for all $Q \in V$ and so $v+\operatorname{ker} V$ is the identity in $A / \operatorname{ker} V$. Hence $J_{x}$ is locally modular, as required.

The next proposition shows part of the connection between local modularity and property (iii) of Proposition 3.2.

Proposition 3.4. Let $A$ be a $C_{0}(X)$-algebra with base map $\phi$ and let $x \in X_{\phi}$. If $J_{x}$ is locally modular then property (iii) of Proposition 3.2 holds, namely for each $b \in \tilde{J}_{x}$ and $\epsilon>0$ there is an open set $V \subseteq \operatorname{Prim}(A)$ with $H(x) \subseteq V$ such that $\|b+\tilde{P}\|<\epsilon$ for all $P \in V$. Hence if $J_{x}$ is locally modular and $\phi$ is locally closed at $x$ then $H_{x}$ is strictly closed.

Proof. Suppose that $J_{x}$ is locally modular and let $b \in \tilde{J}_{x}$ and $\epsilon>0$. Let $P \in \partial H(x)$ and suppose for a contradiction that there is a net $\left(P_{\alpha}\right)$ in $\operatorname{Prim}(A) \backslash U(x)$ such that $P_{\alpha} \rightarrow P$ and $\left\|b+\tilde{P}_{\alpha}\right\| \geq \epsilon$. Then by compactness of the set $W=\{R \in \operatorname{Prim}(M(A)):\|b+R\| \geq \epsilon\}$, and by passing to a subnet of $\left(P_{\alpha}\right)$ if necessary, there exists $R \in W$ such that $\tilde{P}_{\alpha} \rightarrow R$. Since $J_{x}$ is locally modular, it follows from Lemma 3.3 that $R=\tilde{Q}$ for some $Q \in \operatorname{Prim}(A)$. Since $P_{\alpha} \rightarrow Q, \phi(Q)=\phi(P)=x$ and so $b \in \tilde{Q}$, contradicting the fact that $\|b+\tilde{Q}\| \geq \epsilon$. Therefore no such net $\left(P_{\alpha}\right)$ exists. Thus there is an open set $V_{P}$ containing $P$ such that $\|b+\tilde{Q}\|<\epsilon$ for all $Q \in V_{P}$. Taking $V=U(x) \cup \bigcup_{P \in \partial H(x)} V_{P}$ gives the required set $V$.

The final statement now follows from Proposition $3.2(($ ii $)+($ iii $) \Rightarrow($ i $))$.

It will follow from Theorem 3.6 that the converse to Proposition 3.4 holds if $A$ is separable. For a general $\sigma$-unital $C_{0}(X)$-algebra $A$, however, it is possible for property (iii) of Proposition 3.2 to hold for a particular $x \in X_{\phi}$ without $J_{x}$ being locally modular, see Example 4.5. Nevertheless the relation between the two properties is very close, as we shall see.

To show this, we need the following theorem from [7, Theorem 2.5]. For an element $a$ in a $\mathrm{C}^{*}$-algebra $A$, let $\operatorname{sp}(a)$ denote the spectrum of $a$; and for $a \geq 0$, let $\min \operatorname{sp}(a)$ be the smallest number in $\operatorname{sp}(a)$. The function $g$ from the unit interval $[0,1]$ to the space $C[0,1]$ is as follows (where for $r \in[0,1], g_{r}$ is the continuous function on $[0,1]$ corresponding to $r$ ):

$$
\begin{gathered}
\qquad g_{0}(t)=1 \text { for all } t \in[0,1] \\
\text { for } 0<r \leq 1 / 2, \quad g_{r}(t)= \begin{cases}0 & (0 \leq t \leq r / 2) \\
(2 t / r)-1 & (r / 2 \leq t \leq r) \\
1 & (r \leq t \leq 1) ;\end{cases} \\
g_{r}=g_{1 / 2} \text { for } r \geq 1 / 2 .
\end{gathered}
$$

Theorem 3.5. Let $A$ be a $\sigma$-unital $C_{0}(X)$-algebra with base map $\phi$ and set $X_{\phi}=\operatorname{Im}(\phi)$. Let $u$ be a strictly positive element in $A$ with $\|u\|=1$. Let $f \in C^{b}\left(X_{\phi}\right)$ with $0 \leq f \leq 1$, let $U$ be the cozero set of $f$ and let $V=\left\{x \in U: 2 \min \operatorname{sp}\left(u+J_{x}\right) \leq f(x)\right\}$. Let $\operatorname{cl}(U)$ and $\operatorname{cl}(V)$ be the closures of $U$ and $V$ respectively in $X_{\phi}$. Then there exists $b \in M(A)$ with $0 \leq b \leq 1$ such that

(i) $b+\tilde{J}_{x}=g_{f(x)}\left(u+\tilde{J}_{x}\right) \quad\left(x \in X_{\phi}\right)$; 
(ii) $b \in A+H_{x} \subseteq A+\tilde{J}_{x}$ for all $x \in U$;

(iii) $1-b \in \tilde{J}_{x}$ for all $x \in X_{\phi} \backslash U$ and $1-b \in H_{x}$ for all $x \in X_{\phi} \backslash \operatorname{cl}(U)$;

(iv) $\left\|(1-b)+\tilde{J}_{x}\right\|=1$ for all $x \in V$ and $\left\|(1-b)+H_{x}\right\|=1$ for all $x \in \mathrm{cl}(V)$.

Furthermore,

(v) $H_{x}$ is not strictly closed in $M(A)$ for all $x \in \operatorname{cl}(V) \backslash U$.

In the context of Theorem 3.5, note that if $x \in U$ and $A / J_{x}$ is non-unital then $0 \in \operatorname{sp}\left(u+J_{x}\right)$ and hence $x \in V$.

Theorem 3.6. Let $A$ be a $\sigma$-unital $C_{0}(X)$-algebra with base map $\phi$. Let $x \in X_{\phi}$ and let $Z$ be a zero set of $X_{\phi}$ with $x \in Z$. Suppose that $J_{x}$ is not locally modular. Then there exists $y \in Z$ for which property (iii) of Proposition 3.2 fails, that is, for which there exists $c \in \tilde{J}_{y}$ and $\epsilon>0$ such that there is no open set $V \subseteq \operatorname{Prim}(A)$ with $H(y) \subseteq V$ and $\|c+\tilde{P}\|<\epsilon$ for all $P \in V$. In particular, $H_{y}$ is not strictly closed.

Proof. Since $J_{x}$ is not locally modular, it follows from Lemma 3.3 that there exist $P \in \partial H(x)$ and $R \in \operatorname{Prim}(M(A) / A)$ such that $\tilde{P} \sim_{x} R$. Let $D$ be a compact neighbourhood of $P$ in $\operatorname{Prim}(A)$. Let $u \in A$ be a strictly positive element with $\|u\|=1$. Since $u \in A$, it follows that $\|(1-u)+R\|=1$. Hence by $[12,3.3 .2]$, for each $n$ the set

$$
U_{n}=\left\{Q \in \operatorname{Prim}(M(A)):\|(1-u)+Q\|>1-1 / 2^{n+2}\right\}
$$

is an open neighbourhood of $R$ in $\operatorname{Prim}(M(A))$. Let $h: X_{\phi} \rightarrow[0,1]$ be a continuous function such that $Z(h)=Z$ and, for $n \geq 1$, let $V_{n}=\{Q \in \operatorname{Prim}(A): h(\phi(Q))<1 / n\}$. Then $V_{n}$ is an open neighbourhood of $P$ in $\operatorname{Prim}(A)$. Since $\tilde{P} \sim_{x} R$, it follows that

$$
U_{1} \cap \tilde{V}_{1} \cap(\operatorname{Int} D)^{\sim} \cap(\operatorname{Prim}(A) \backslash U(x))^{\sim}
$$

is a non-empty, relatively open, subset of $(\operatorname{Prim}(A) \backslash U(x))^{\sim}$.

Since $\operatorname{Prim}(A) \backslash H(x)$ is dense in $\operatorname{Prim}(A) \backslash U(x)$, we may choose $Q_{1} \in \operatorname{Prim}(A) \backslash H(x)$ with $\tilde{Q}_{1} \in U_{1} \cap \tilde{V}_{1} \cap(\operatorname{Int} D)^{\sim}$. Set $x_{1}=\phi\left(Q_{1}\right)$. Then $x_{1} \neq x$ and so there exists a continuous function $f_{1}: X_{\phi} \rightarrow[0,1 / 4]$ with $f_{1}\left(x_{1}\right)=1 / 4$ and $f_{1}(x)=0$. For $n \geq 2$, we will inductively define points $x_{n} \in X_{\phi}$ and continuous functions $f_{n}: X_{\phi} \rightarrow\left[0,1 / 2^{n+1}\right]$ with $f_{n}\left(x_{n}\right)=1 / 2^{n+1}$ and $f_{n}(x)=0=f_{n}\left(x_{m}\right)$ for $1 \leq m \leq n-1$. Note that $x_{1}$ and $f_{1}$ satisfy these conditions.

Suppose that $n \geq 2$ and that $x_{1}, \ldots, x_{n-1}$ and $f_{1}, \ldots, f_{n-1}$ satisfy the required conditions. Let $W_{n}=\left\{Q \in \operatorname{Prim}(A): \sum_{i=1}^{n-1} f_{i}(\phi(Q))<1 / 2^{n+1}\right\}$. Then $W_{n}$ is an open neighbourhood of $P$ and hence, since $\tilde{P} \sim_{x} R$, it follows that

$$
U_{n} \cap \tilde{V}_{n} \cap(\operatorname{Int} D)^{\sim} \cap \tilde{W}_{n} \cap(\operatorname{Prim}(A) \backslash U(x))^{\sim}
$$

is a non-empty, relatively open, subset of $(\operatorname{Prim}(A) \backslash U(x))^{\sim}$. Thus we may choose $Q_{n} \in$ $\operatorname{Prim}(A) \backslash H(x)$ with $\tilde{Q}_{n} \in U_{n} \cap \tilde{V}_{n} \cap(\operatorname{Int} D)^{\sim} \cap \tilde{W}_{n}$. Set $x_{n}=\phi\left(Q_{n}\right)$. Then $x_{n} \neq x$ and $x_{n} \neq x_{m}$ for $1 \leq m \leq n-1$ because

$$
\sum_{i=1}^{n-1} f_{i}\left(x_{m}\right) \geq f_{m}\left(x_{m}\right)=\frac{1}{2^{m+1}}>\frac{1}{2^{n+1}}>\sum_{i=1}^{n-1} f_{i}\left(x_{n}\right) .
$$

Thus there exists a continuous function $f_{n}: X_{\phi} \rightarrow\left[0,1 / 2^{n+1}\right]$ with $f_{n}\left(x_{n}\right)=1 / 2^{n+1}$ and $f_{n}(x)=0=f_{n}\left(x_{m}\right)$ for $1 \leq m \leq n-1$. 
Set $f=\sum_{n=1}^{\infty} f_{n}$. Then $f$ is continuous and, for each $n \geq 1$,

$$
\frac{1}{2^{n+1}}=f_{n}\left(x_{n}\right) \leq f\left(x_{n}\right)=\sum_{i=1}^{n-1} f_{i}\left(x_{n}\right)+f_{n}\left(x_{n}\right)<\frac{1}{2^{n+1}}+\frac{1}{2^{n+1}}=\frac{1}{2^{n}} \text {. }
$$

Thus $f\left(x_{n}\right) \rightarrow 0$ as $n \rightarrow \infty$. Furthermore, since $\left\|(1-u)+\tilde{Q}_{n}\right\|>1-1 / 2^{n+2}$, it follows that

$$
\min \operatorname{sp}\left(u+\tilde{Q}_{n}\right)<\frac{1}{2^{n+2}} \leq \frac{f\left(x_{n}\right)}{2}
$$

and hence $0 \in \operatorname{sp}\left(g_{f\left(x_{n}\right)}\left(u+\tilde{Q}_{n}\right)\right)$ for $n \geq 1$.

Since $Q_{n} \in D$ for all $n$, the compactness of $D$ implies that there exists $Q \in D$ and a subnet $\left(Q_{n_{\alpha}}\right)$ of $\left(Q_{n}\right)$ such that $Q_{n_{\alpha}} \rightarrow Q$. Set $y=\phi(Q)$. Since $Q_{n} \in V_{n}(n \geq 1), h\left(x_{n}\right) \rightarrow 0$ as $n \rightarrow \infty$. It follows that $h(y)=0$ and hence $y \in Z$. Furthermore, $f\left(x_{n_{\alpha}}\right) \rightarrow f(y)$ and so $f(y)=0$. Let $b$ be an element of $M(A)$ corresponding to $f$ as in Theorem 3.5. Then $1-b \in \tilde{J}_{y}$ by Theorem 3.5(iii). Since $\phi\left(Q_{n}\right)=x_{n}(n \geq 1)$, it follows that $\tilde{Q}_{n} \supseteq \tilde{J}_{x_{n}}$ and hence that $b+\tilde{Q}_{n}=g_{f\left(x_{n}\right)}\left(u+\tilde{Q}_{n}\right)$ by Theorem 3.5(i). Then

$$
1 \geq\left\|(1-b)+\tilde{Q}_{n}\right\| \geq\left\|\left(1+\tilde{Q}_{n}\right)-g_{f\left(x_{n}\right)}\left(u+\tilde{Q}_{n}\right)\right\|=1-\min \operatorname{sp}\left(g_{f\left(x_{n}\right)}\left(u+\tilde{Q}_{n}\right)\right)=1 .
$$

Hence $\left\|(1-b)+\tilde{Q}_{n_{\alpha}}\right\|=1$ for all $\alpha$. Suppose that $V$ is an open subset of $\operatorname{Prim}(A)$ such that $H(y) \subseteq V$. Then $Q \in V$ and so eventually $Q_{n_{\alpha}} \in V$. Thus property (iii) of Proposition 3.2 fails at $y$ for $c=1-b \in \tilde{J}_{y}$ and $\epsilon=1 / 2$. Hence $H_{y}$ is not strictly closed by Proposition 3.2.

Regarding the hypotheses of Theorem 3.6, we note that the complete regularity of $X_{\phi}$ implies that every neighbourhood of $x$ contains a zero set containing $x$. In the proof of Theorem 3.6, we could have checked that $y \in \operatorname{cl}(V) \backslash U$ (in the terminology of Theorem 3.5) and used Theorem 3.5(v) to deduce that $H_{y}$ is not strictly closed. We have preferred, however, to obtain the stronger result that property (iii) of Proposition 3.2 fails at $y$.

Armed with Theorem 3.6, we can now prove some of the main results of the paper.

Corollary 3.7 (global spectral synthesis). Let $A$ be a $C_{0}(X)$-algebra with base map $\phi$. Consider the following two properties.

(i) $H_{x}$ is strictly closed for all $x \in X_{\phi}$.

(ii) $J_{x}$ is locally modular and $\phi$ is locally closed at $x$ for all $x \in X_{\phi}$.

Then (ii) $\Rightarrow(\mathrm{i})$, and (i) and (ii) are equivalent if $A$ is $\sigma$-unital.

Proof. (ii) $\Rightarrow$ (i). This follows from Proposition 3.4.

(i) $\Rightarrow$ (ii) (assuming that $A$ is $\sigma$-unital). This follows from (i) $\Rightarrow(($ ii $)+($ iii $)$ ) of Proposition 3.2 together with Theorem 3.6 (taking $Z=X_{\phi}$, for example).

Corollary 3.8 (spectral synthesis at a point). Let $A$ be a $\sigma$-unital $C_{0}(X)$-algebra with base map $\phi$ and let $x$ be a $G_{\delta}$-point in $X_{\phi}$. Then $H_{x}$ is strictly closed if and only if $J_{x}$ is locally modular and $\phi$ is locally closed at $x$.

Proof. This follows from Proposition 3.4 and from Proposition 3.2 and Theorem 3.6 taking the zero set $Z$ in Theorem 3.6 to be $Z=\{x\}$.

Recall that a completely regular (Hausdorff) space $X$ is perfectly normal if every closed subset of $X$ is the zero set of a continuous real-valued function on $X$. Every metric space is perfectly normal. 
Lemma 3.9. Let $A$ be a separable $C_{0}(X)$-algebra with base map $\phi$. Then $X_{\phi}$ is perfectly normal.

Proof. Let $V$ be an open subset of $X_{\phi}$. Set $Y=\phi^{-1}(V)$ and let $J$ be the ideal of $A$ such that $\operatorname{Prim}(J)$ is canonically homeomorphic to $Y$. Then $J$ is separable. Let $u$ be a strictly positive element in $J$. For each $n \in \mathbf{N}$, set $Y_{n}=\{P \in \operatorname{Prim}(A):\|u+P\| \geq 1 / n\}$. Then $Y_{n}$ is compact and so $\phi\left(Y_{n}\right)$ is a compact (hence closed) subset of $X_{\phi}$. Since $V=\bigcup_{n=1}^{\infty} \phi\left(Y_{n}\right)$, we see that $V$ is an $F_{\sigma}$ subset of $X_{\phi}$. But $X_{\phi}$ is normal, and in a normal space an open $F_{\sigma}$ subset is a cozero set. Thus $V$ is a cozero set.

Corollary 3.10. Let $A$ be a separable $C_{0}(X)$-algebra with base map $\phi$ and let $x \in X_{\phi}$. Then $H_{x}$ is strictly closed if and only if $J_{x}$ is locally modular and $\phi$ is locally closed at $x$.

Proof. This follows as for Corollary 3.8, noting that $\{x\}$ is a zero set of $X_{\phi}$ for each $x \in X_{\phi}$ by Lemma 3.9 .

\section{Pointwise spectral synthesis and P-Points}

Recall from Section 2 that if $A$ is a $C_{0}(X)$-algebra with base map $\phi$ then $U_{\phi}=\left\{x \in X_{\phi}\right.$ : $\left.J_{x} \nsupseteq Z^{\prime}(A)\right\}$, an open subset of $X_{\phi}, \partial U_{\phi}$ denotes the boundary of $U_{\phi}$ in $X_{\phi}$ and $W_{\phi}=X_{\phi} \backslash U_{\phi}$. Here $Z^{\prime}(A)=\mu\left(C_{0}(X)\right) \cap A$. We saw in Proposition 2.6 that if $x \in U_{\phi}$ or if $x$ is an isolated point of $X_{\phi}$ then $H_{x}$ is strictly closed, while if $A / J_{x}$ is unital but $x \notin U_{\phi}$ then $H_{x}$ is not strictly closed. We remarked that the remaining points to consider are those for which $A / J_{x}$ is non-unital and $x \in W_{\phi}$ and is non-isolated in $X_{\phi}$. From Section 3 we now have a complete characterization of global spectral synthesis for $\sigma$-unital $A$, and a complete characterization of pointwise spectral synthesis for separable $A$, but only a near-characterization of pointwise spectral synthesis for the more general case when $A$ is $\sigma$-unital.

In this section we approach the $\sigma$-unital case from another angle. We characterize the points $x$ in the interior of $W_{\phi}$ in $X_{\phi}$ for which $H_{x}$ is closed (these turn out to be precisely the P-points) and make partial progress for the difficult case of points $x \in \partial U_{\phi}$ (cf. for example [6, Proposition 3.5]). We also give a complete characterization of pointwise spectral synthesis for the important case when the base map $\phi$ is open (Theorem 4.6). We begin with the following lemma.

Lemma 4.1. Let $A$ be a $\sigma$-unital $C_{0}(X)$-algebra with base map $\phi$ and let $x \in W_{\phi}$. Let $u \in A$ be strictly positive with $\|u\| \leq 1$. Then there is a net $\left(x_{\alpha}\right)$ in $X_{\phi}$ with $x_{\alpha} \rightarrow x$ and $\left\|(1-u)+\tilde{J}_{x_{\alpha}}\right\| \rightarrow 1$.

Proof. By Lemma 2.5 there exists $R \in \operatorname{Prim}(M(A) / A)$ with $R \supseteq H_{x}$. Let $\left(P_{\alpha}\right)$ be a net in $\operatorname{Prim}(A)$ such that $\tilde{P}_{\alpha} \rightarrow R$. Then $\bar{\phi}\left(\tilde{P}_{\alpha}\right) \rightarrow \bar{\phi}(R)=x$. Hence $x_{\alpha}:=\phi\left(P_{\alpha}\right) \rightarrow x$. On the other hand, since $R \supseteq A$,

$$
\begin{aligned}
1=\|(1-u)+R\| & \leq \liminf \left\|(1-u)+\tilde{P}_{\alpha}\right\| \\
& \leq \liminf \left\|(1-u)+\tilde{J}_{x_{\alpha}}\right\| \\
& \leq \limsup \left\|(1-u)+\tilde{J}_{x_{\alpha}}\right\| \leq 1 .
\end{aligned}
$$

Hence $\left\|(1-u)+\tilde{J}_{x_{\alpha}}\right\| \rightarrow 1$.

Next we recall the definition of a P-point. Let $X$ be a completely regular (Hausdorff) space. A point $x \in X$ is a $P$-point if every continuous real-valued function vanishing at $x$ 
vanishes in a neighbourhood of $x[18,4 \mathrm{~L}]$. Equivalently, $x$ is a P-point if $x$ does not lie in the boundary of any cozero set. If the space $X$ is perfectly normal then every singleton is a zero set and so a P-point is necessarily an isolated point. A space in which every point is a $\mathrm{P}$-point is a $P$-space.

We are now ready for the first main result of this section.

Theorem 4.2. Let $A$ be a $\sigma$-unital $C_{0}(X)$-algebra with base map $\phi$. If $x \in X_{\phi}$ is a P-point in $X_{\phi}$ then $H_{x}$ is strictly closed. Conversely, if $x \in W_{\phi}$ and $H_{x}$ is strictly closed then $x$ is a P-point in $W_{\phi}$.

Proof. Let $x \in X_{\phi}$ and suppose that $H_{x}$ is not strictly closed. We show that $x$ is not a Ppoint in $X_{\phi}$. By [21, Theorem 10.1.7], there exists $b \in \tilde{J}_{x} \backslash H_{x}$ with $\|b\|=\left\|b+H_{x}\right\|=1$. Let $u$ be a strictly positive element of $A$ with $\|u\|=1$, and recall that for $y \in X_{\phi}, b \in \tilde{J}_{y}$ if and only if $b u \in \widetilde{J}_{y}$ (cf. [6, Section 2]). For each $n \geq 1$, set $W_{n}=\left\{y \in X_{\phi}:\left\|b u+\tilde{J}_{y}\right\| \geq 1 / n\right\}$. By [7, Lemma 4.2], for every neighbourhood $W$ of $x$ in $\operatorname{cl}_{\beta X} X_{\phi}$,

$$
\left\|b+H_{x}\right\| \leq \sup \left\{\left\|b+\tilde{J}_{y}\right\|: y \in X_{\phi} \cap W\right\} .
$$

Hence for every neighbourhood $V$ of $x$ in $X_{\phi}$ there exists $y \in V$ such that $b u \notin \tilde{J}_{y}$. Thus $x$ lies in the closure of $\bigcup_{n=1}^{\infty} W_{n}$. Since

$$
W_{n}=\left\{y \in X_{\phi}:\left\|b u+J_{y}\right\| \geq 1 / n\right\}=\phi(\{P \in \operatorname{Prim}(A):\|b u+P\| \geq 1 / n\}),
$$

$W_{n}$ is a compact subset of $X_{\phi}$. But $x \notin W_{n}$ and hence there exists a continuous function $f_{n}: X_{\phi} \rightarrow[0,1]$ such that $f_{n}(x)=0$ and $f_{n}\left(W_{n}\right)=\{1\}$. Set $f=\sum_{n=1}^{\infty} f_{n} / 2^{n}$. Then $f(x)=0$, but $x$ lies in the closure of the cozero set of $f$. Hence $x$ is not a P-point.

Conversely, suppose that $x \in W_{\phi}$ is not a P-point in $W_{\phi}$. Let $f$ be a continuous function on $W_{\phi}$ with $f(x)=0$ such that $x$ lies in the closure of the cozero set of $f$. Replacing $f$ by $\min \{|f|, 1\}$, we may assume that $0 \leq f \leq 1$. Since $X_{\phi}$ is normal and $W_{\phi}$ is closed in $X_{\phi}$, we may extend $f$ to a continuous function $\overline{\bar{f}}$ on $X_{\phi}$ with $0 \leq \bar{f} \leq 1$. Let $y \in W_{\phi} \cap \operatorname{coz}(\bar{f})$. By Lemma 4.1 there is a net $\left(y_{\alpha}\right)$ in $X_{\phi}$ with $y_{\alpha} \rightarrow y$ and $\left\|(1-u)+\tilde{J}_{y_{\alpha}}\right\| \rightarrow 1$. Hence eventually $2 \min \operatorname{sp}\left(u+J_{y_{\alpha}}\right)=2\left(1-\left\|(1-u)+\tilde{J}_{y_{\alpha}}\right\|\right) \leq \bar{f}\left(y_{\alpha}\right)$, since $\bar{f}\left(y_{\alpha}\right) \rightarrow \bar{f}(y)>0$. It follows that the set $V$ of Theorem 3.5 (associated with the cozero set $\operatorname{coz}(\bar{f})$ ) has closure $\operatorname{cl}(V)$ containing $W_{\phi} \cap \operatorname{coz}(\bar{f})$. Hence $x \in \operatorname{cl}(V)$ since $x$ lies in the the closure of $W_{\phi} \cap \operatorname{coz}(\bar{f})=\operatorname{coz}(f)$. Thus $\tilde{J}_{x} \neq H_{x}$ by Theorem $3.5(\mathrm{v})$.

Theorem 4.2 has some useful consequences. Let Int $W_{\phi}$ denote the interior of $W_{\phi}$ relative to $X_{\phi}$.

Corollary 4.3. Let $A$ be a $C_{0}(X)$-algebra with base map $\phi$ and let $x \in \operatorname{Int} W_{\phi}$. If $A$ is $\sigma$-unital then $H_{x}$ is strictly closed if and only if $x$ is a P-point in $X_{\phi}$. If $A$ is separable then $H_{x}$ is strictly closed if and only if $x$ is an isolated point in $X_{\phi}$.

Proof. Suppose that $A$ is $\sigma$-unital and that $x$ is not a P-point in $X_{\phi}$. Then there exists $f \in C^{b}\left(X_{\phi}\right)$ such that $f(x)=0$ and $x$ lies in the closure of $\operatorname{coz}(f)$. Let $\left(x_{\alpha}\right)$ be a net in $\operatorname{coz}(f)$ such that $x_{\alpha} \rightarrow x$, and set $\bar{f}=\left.f\right|_{W_{\phi}}$. Then $\bar{f}$ is continuous and $\bar{f}(x)=0$ but eventually $x_{\alpha} \in$ Int $W_{\phi}$ and so $x$ lies in the closure of the cozero set of $\bar{f}$. Thus $x$ is not a P-point in $W_{\phi}$ and so $H_{x}$ is not strictly closed by Theorem 4.2. If $A$ is separable then $\{x\}$ is a zero set in $X_{\phi}$ by Lemma 3.9 and hence $x$ is $\mathrm{P}$-point in $X_{\phi}$ if and only if it is isolated in $X_{\phi}$. 
Corollary 4.4. Let $A$ be a $C_{0}(X)$-algebra with base map $\phi$ and suppose that $Z^{\prime}(A)=\{0\}$.

(i) Let $x \in X_{\phi}$. If $A$ is $\sigma$-unital then $H_{x}$ is strictly closed if and only if $x$ is a P-point in $X_{\phi}$. If $A$ is separable then $H_{x}$ is strictly closed if and only if $x$ is an isolated point in $X_{\phi}$.

(ii) If $A$ is $\sigma$-unital then $H_{x}$ is strictly closed for all $x \in X_{\phi}$ if and only if $X_{\phi}$ is discrete.

Proof. Since $Z^{\prime}(A)=\{0\}, U_{\phi}=\emptyset$ and $W_{\phi}=X_{\phi}$. Thus part (i) follows from Corollary 4.3. Part (ii) now follows because the space $X_{\phi}$ is $\sigma$-compact, and a $\sigma$-compact P-space is discrete (see, for example, the proof of [7, Lemma 4.4]).

If $A$ is a stable $C_{0}(X)$-algebra then $Z(A)=\{0\}$ and hence $Z^{\prime}(A)=\{0\}$. Thus Corollary 4.4 extends [7, Theorem 4.5].

It follows from Theorem 4.2 and Proposition 3.2 that if $A$ is a $\sigma$-unital $C_{0}(X)$-algebra and $x \in X_{\phi}$ is a $\mathrm{P}$-point in $X_{\phi}$ then $\phi$ is locally closed at $x$ and that property (iii) of Proposition 3.2 holds. On the other hand, $J_{x}$ need not be locally modular as the following example shows.

Example 4.5. Let $X$ be a compact Hausdorff space with a non-isolated P-point $x$ (e.g. take $X$ to be $\omega_{1}+1$, where $\omega_{1}$ is the first uncountable ordinal, with the usual topology) and let $A=C(X) \otimes K(H)$ (where $K(H)$ is the algebra of compact operators on a separable, infinite-dimensional Hilbert space $H)$. There is a homeomorphism $\phi: \operatorname{Prim}(A) \rightarrow X$ such that

$$
\phi\left(\left\{f \in C_{0}(X): f(y)=0\right\} \otimes K(H)\right)=y \quad(y \in X) .
$$

Then $J_{x}$ is not locally modular because $\partial H(x)$ is non-empty but $A / P$ is non-unital for all $P \in \operatorname{Prim}(A)$. Nevertheless, $H_{x}$ is strictly closed by Theorem 4.2 .

In Theorem 4.2 we saw a characterization, for $A \sigma$-unital, of when $H_{x}$ is strictly closed for $x \in$ Int $W_{\phi}$ and we know that $H_{x}$ is always strictly closed if $x \in U_{\phi}$ (Proposition 2.6(i)). The remaining points to consider are those in $\partial U_{\phi}$. For general $\sigma$-unital $C_{0}(X)$-algebras we are not able to characterize the points $x \in \partial U_{\phi}$ for which $H_{x}$ is strictly closed (though we have seen a necessary condition in Theorem 4.2), but if we make the further assumption that the base map $\phi$ is open then we can show that there are no such points.

Theorem 4.6. Let $A$ be a continuous, $\sigma$-unital $C_{0}(X)$-algebra with base map $\phi$ and let $x \in X_{\phi}$. Then the following are equivalent:

(i) $H_{x}$ is strictly closed;

(ii) either $x \in U_{\phi}$, or $x \in \operatorname{Int} W_{\phi}$ and $x$ is a P-point in $X_{\phi}$.

Proof. (ii) $\Rightarrow$ (i) If $x \in U_{\phi}$, or if $x \in \operatorname{Int} W_{\phi}$ with $x$ a P-point in $X_{\phi}$, then $H_{x}$ is strictly closed by Proposition 2.6(i) and Corollary 4.3.

(i) $\Rightarrow$ (ii) Corollary 4.3 shows that if $x \in$ Int $W_{\phi}$ with $H_{x}$ strictly closed then $x$ must be a P-point in $X_{\phi}$. It is enough, therefore, to show that if $x \in \partial U_{\phi}$ then $H_{x}$ is not strictly closed. If $A / J_{x}$ is unital then this follows from Proposition 2.6(iii). Hence we may assume that $A / J_{x}$ is non-unital. Let $u$ be a strictly positive element of $A$ with $\|u\|=1$. Then $\left\|(1-u)+\tilde{J}_{x}\right\|=1$. For each $n \geq 1$, there exists $P_{n} \in \operatorname{Prim}\left(A / J_{x}\right)$ such that $\left\|(1-u)+\tilde{P}_{n}\right\|>1-1 / 2^{n+1}$. Hence the set

$$
V_{n}=\left\{Q \in \operatorname{Prim}(A):\|(1-u)+\tilde{Q}\|>1-1 / 2^{n+1}\right\}
$$

is an open neighbourhood of $P_{n}$ and so, since $\phi$ is open, the set $\phi\left(V_{n}\right)$ is an open neighbourhood of $\phi\left(P_{n}\right)=x$ in $X_{\phi}$. Thus

$$
X_{n}:=X_{\phi} \backslash \phi\left(V_{n}\right)=\left\{y \in X_{\phi}:\left\|(1-u)+\tilde{J}_{y}\right\| \leq 1-1 / 2^{n+1}\right\}
$$


is closed in $X_{\phi}$ and $x \notin X_{n}$. If $y \in U_{\phi}$ then $A / J_{y}$ is unital [6, Proposition 2.2] and therefore $\left\|(1-u)+\tilde{J}_{y}\right\|<1$. Hence $\bigcup_{n=1}^{\infty} X_{n} \supseteq U_{\phi}$ and it follows that $x$ lies in the closure of $\left(\bigcup_{n=2}^{\infty} X_{n}\right) \backslash X_{1}$.

Since $x \notin X_{n}$ there exists $f_{n} \in C^{b}\left(X_{\phi}\right)$ with $0 \leq f_{n} \leq 1 / 2^{n}$ such that $f_{n}(x)=0$ and $f_{n}\left(X_{n}\right)=\left\{1 / 2^{n}\right\}$. Set $f=\sum_{n=1}^{\infty} f_{n}$. Then $f \in C^{b}\left(X_{\phi}\right)$ with $0 \leq f \leq 1$ and $f(x)=0$. Let $W$ be the cozero set of $f$ and let $V=\left\{y \in W: 2 \min \operatorname{sp}\left(u+J_{y}\right) \leq f(y)\right\}$. Suppose that $y \in X_{\phi}$ with $y \in X_{n+1} \backslash X_{n}$ for some $n \geq 1$. Then $\left\|(1-u)+\tilde{J}_{y}\right\|>1-1 / 2^{n+1}$ and so $\min \operatorname{sp}\left(u+J_{y}\right)<1 / 2^{n+1}$. Since $y \in X_{m}$ for all $m \geq n+1, f(y) \geq 1 / 2^{n}$ and so $f(y)>2 \min \operatorname{sp}\left(u+J_{y}\right)$. Hence $y \in V$, and thus $V \supseteq\left(\bigcup_{n=2}^{\infty} X_{n}\right) \backslash X_{1}$. Hence $x \in \operatorname{cl}(V) \backslash W$ and so it follows from Theorem $3.5(\mathrm{v})$ that $\tilde{J}_{x} \neq H_{x}$, as required.

Corollary 4.7. Let $A$ be a continuous, $\sigma$-unital $C_{0}(X)$-algebra with base map $\phi$. Then the following are equivalent:

(i) for all $x \in X_{\phi}, H_{x}$ is strictly closed;

(ii) $U_{\phi}$ and $W_{\phi}$ are clopen in $X_{\phi}$, and $W_{\phi}$ is discrete.

Proof. (ii) $\Rightarrow$ (i). This follows immediately from Theorem 4.6.

$(\mathrm{i}) \Rightarrow(\mathrm{ii})$. Theorem 4.6 implies that $\partial U_{\phi}$ is empty and hence $X_{\phi}$ has the required decomposition into clopen sets $U_{\phi}$ and $W_{\phi}$. Since $X_{\phi}$ is $\sigma$-compact, $W_{\phi}$ must be $\sigma$-compact as well. But by Theorem 4.6, $W_{\phi}$ is a $\mathrm{P}$-space, and a $\sigma$-compact $\mathrm{P}$-space is discrete, see [7, Lemma 4.4].

It is interesting to compare Corollary 4.7 with [6, Theorem 3.8] which characterizes, for a continuous $\sigma$-unital $C_{0}(X)$-algebra $A$, when $M(A)$ is a continuous $C(\beta X)$-algebra. The conditions in Corollary 4.7 are markedly stronger than those in [6, Theorem 3.8], notably the requirement that $W_{\phi}$ be discrete as against being a basically disconnected space. On the other hand, if $A$ is separable then there is a much closer fit with [6, Corollary 3.9]: indeed, if $A$ is continuous and separable and $X_{\phi}=X$ then $H_{x}$ is strictly closed for all $x \in X_{\phi}$ if and only if $M(A)$ is continuous for $\bar{\mu}$.

We conclude this section with a couple of elementary abelian examples, part of whose significance will appear in the next two sections.

Example 4.8. (i) An abelian $C_{0}(X)$-algebra $A$ with $x \in W_{\phi}$ such that $H_{x}$ is strictly closed in $M(A)$. Let $Y=\left\{(x, y) \in \mathbf{R}^{2}: y \geq 0\right\}$ be the upper half-plane, and let $L=\{(x, y) \in$ $Y: y=0\}$ be the $x$-axis. Let $Y / L$ be the quotient space (which is completely regular by Lemma 2.8). Set $A=C_{0}(Y)$. Then we may identify $\operatorname{Prim}(A)$ with $Y$ in the usual way and define $\phi: \operatorname{Prim}(A) \rightarrow \beta(Y / L)$ by $\phi((x, y))=[(x, y)] \in Y / L$. Thus $X_{\phi}=Y / L$. Then $J_{[(0,0)]}$ is locally modular (since every point in $L$ has a compact neighbourhood in $Y$ ) and $\phi$ is locally closed at $[(0,0)]$ (since $Y$ is normal). However, $[(0,0)] \notin U_{\phi}$ because if $f \in C(\beta Y / L)$ and $f \circ \phi \in A=C_{0}(Y)$ then $f([(0,0)])=0$.

(ii) An abelian $C_{0}(X)$-algebra $A$ with $x \in X_{\phi}$ such that $H_{x}$ is not strictly closed in $M(A)$. Let $A=C_{0}([0,1) \cup[2,3])$ and set $X=[0,1]$. Then we may identify $\operatorname{Prim}(A)$ with $[0,1) \cup[2,3]$. Let $\phi: \operatorname{Prim}(A) \rightarrow X$ be given by $\phi(x)=x(0 \leq x<1)$ and $\phi(x)=1(2 \leq x \leq 3)$. Then $J_{x}$ is locally modular for all $x \in X$, but $\phi$ is not locally closed at $x=1$. Hence $H_{1}$ is not strictly closed in $M(A)$. 


\section{5. $C_{0}(X)$-Algebras WHERE THE BASE MAP $\phi$ IS THE COMPLETE REgularization MaP}

In this section we investigate $C_{0}(X)$-algebras $A$ where the base map $\phi$ is the complete regularization map on $\operatorname{Prim}(A)$ [18, Theorem 3.9]. Thus $X$ may be taken to be the complete regularization space (in cases where this is locally compact) or its Stone-Čech compactification. Restricting $\phi$ in this way places a considerable constraint on its behaviour, as we shall see. This case is of special interest for two reasons: firstly, the complete regularization map interacts with the topology on $\operatorname{Prim}(A)$ in a way that is lacking with more general continuous maps, and secondly, every continuous map from $\operatorname{Prim}(A)$ to a locally compact Hausdorff space factors through the complete regularization map.

Under the hypotheses that $\phi$ is the complete regularization map and that $\operatorname{Orc}(A)<\infty$ (a technical assumption which is usually satisfied), we show that if $A$ is $\sigma$-unital and $J_{x}$ is locally modular then $\phi$ is locally closed at $x$ and $H_{x}$ is strictly closed (Theorem 5.3). Thus the 'locally closed at $x$ ' part of the hypothesis in the final sentence of Proposition 3.4 is automatically satisfied in this case (contrast with Example 4.8(ii)).

We begin by explaining the notation $\operatorname{Orc}(A)$ [33]. Recall that for a $\mathrm{C}^{*}$-algebra $A$ and for $P, Q \in \operatorname{Prim}(A)$ we write $P \sim Q$ if $P$ and $Q$ cannot be separated by disjoint open sets in $\operatorname{Prim}(A)$. The relation $\sim$ on $\operatorname{Prim}(A)$ induces a graph structure on $\operatorname{Prim}(A)$ whereby $P$ and $Q$ are adjacent if $P \sim Q$. The distance $d_{A}(P, Q)$ between $P$ and $Q$ is then defined as the length of the shortest path from $P$ to $Q$ (and is $\infty$ if no such path exists). The diameter of a $\sim$-component of $\operatorname{Prim}(A)$ is the supremum of the distances between primitive ideals in the component (with the convention that a singleton component, such as when $\operatorname{Prim}(A)$ is Hausdorff, has diameter 1). The connecting order, $\operatorname{Orc}(A)$, is the supremum of the diameters of $\sim$-components of $\operatorname{Prim}(A)$. Clearly $\operatorname{Orc}(A)$ is an integer between 1 and $\infty$, and all possibilities occur, including $\infty$ [33, Example 2.8] (see also Example 6.4(ii) below). The smaller that $\operatorname{Orc}(A)$ is, the nearer $\operatorname{Prim}(A)$ is to being Hausdorff, with the case $\operatorname{Orc}(A)=1$ corresponding to $\sim$ being an equivalence relation on $\operatorname{Prim}(A)$. For a subset $Y \subseteq \operatorname{Prim}(A)$ and for $n \geq 0$, let $Y^{n}=\left\{P \in \operatorname{Prim}(A): \exists Q \in Y, d_{A}(P, Q) \leq n\right\}$.

We also need the following topological lemma characterizing separation by open sets. We say that a topological space is locally compact if every point has a neighbourhood base of compact sets.

Lemma 5.1. Let $X$ be a locally compact topological space and let $Y$ and $Z$ be subsets of $X$ which are Lindelof in the relative topology. Then the following are equivalent:

(i) The closure of $Y^{1}$ does not meet $Z$ and the closure of $Z^{1}$ does not meet $Y$.

(ii) There exist disjoint open subsets $U$ and $V$ of $X$ with $Y \subseteq U$ and $Z \subseteq V$.

Proof. Suppose first that (ii) holds. Then $X \backslash V$ is disjoint from $Z$ and is a closed set containing the neighbourhood $U$ of $Y$ and hence containing $Y^{1}$. Similarly $X \backslash U$ is disjoint from $Y$ and is a closed set containing $Z^{1}$. Hence (i) holds.

Conversely, suppose that (i) holds. Let $x \in Y$. Since the closure of $Z^{1}$ does not meet $Y$, $x$ has an open neighbourhood disjoint from $Z^{1}$. Hence, by the local compactness of $X, x$ has a compact neighbourhood $U_{x} \subseteq X \backslash Z^{1}$. Then $U_{x}^{1}$ is closed because $U_{x}$ is compact, and $U_{x}^{1}$ does not meet $Z$ because $U_{x}$ does not meet $Z^{1}$. Similarly for each $x \in Z$ there exists a neighbourhood $V_{x}$ of $x$ such that the closure of $V_{x}$ does not meet $Y$.

Since $Y$ and $Z$ are Lindelof, we may obtain a countable collection, say $U_{1}, U_{2}, \ldots$, of the sets Int $U_{x}$ such that $\bigcup_{i=1}^{\infty} U_{i}$ covers $Y$, and likewise a countable collection $V_{1}, V_{2}, \ldots$ 
of the sets Int $V_{x}$ such that $\bigcup_{i=1}^{\infty} V_{i} \operatorname{covers} Z$. For each $i \geq 1$, set $U_{i}^{\prime}=U_{i} \backslash\left(\bigcup_{j=1}^{i} \bar{V}_{j}\right)$ and $V_{i}^{\prime}=V_{i} \backslash\left(\bigcup_{j=1}^{i} \bar{U}_{j}\right)$ (where $\bar{V}_{i}$ denotes the closure of $V_{i}$, etc). Set $U=\bigcup_{i=1}^{\infty} U_{i}^{\prime}$ and $V=\bigcup_{i=1}^{\infty} V_{i}^{\prime}$. Then it is easily checked that $Y \subseteq U$ and $Z \subseteq V$. If $x \in U \cap V$ then there exist $U_{i}^{\prime}$ and $V_{j}^{\prime}$ such that $x \in U_{i}^{\prime} \cap V_{j}^{\prime}$. Without loss of generality we may suppose that $i \geq j$. But then $V_{j}^{\prime} \subseteq V_{j}$ which is disjoint from $U_{i}^{\prime}$, a contradiction. Hence $U$ and $V$ are disjoint, and thus (ii) holds.

Now let $A$ be a $\sigma$-unital $C_{0}(X)$-algebra. Let $E$ be a non-empty closed subset of $X_{\phi}$, set $Y=X_{\phi} / E$ and let $q: X_{\phi} \rightarrow Y$ be the quotient map. Set $\psi=q \circ \phi$ and $\{e\}=q(E)$. We saw in Proposition 2.9 that the question of spectral synthesis for the set $E$ can be reduced to that of the point $e$, and for this reason we have previously confined ourselves to considering spectral synthesis at points. If we restrict $\phi$ to be the complete regularization map, however, then we can no longer make this reduction (because the reduction changes the base map), and we will therefore have to work with closed sets in this section.

With this in mind, and with the notation above, we say that $\phi$ is locally closed at $E$ if $\psi$ is locally closed at $e$, and that $J_{E}$ is locally modular if $J_{e}$ is locally modular. Elementary topological arguments show that $\phi$ is locally closed at $E$ if and only if whenever $Y$ is a closed subset of $\operatorname{Prim}(A)$ such that $\phi(Y) \cap E=\emptyset$ (i.e. $Y \cap \phi^{-1}(E)=\emptyset$ ) then $\overline{\phi(Y)} \cap E=\emptyset$. Note that $H(e)=(q \circ \phi)^{-1}(e)=\phi^{-1}(E)$, the hull of $J_{E}$ in Prim(A), and recall that $U(e)$ is the interior of $H(e)$ in $\operatorname{Prim}(A)$. For $Q, R \in \operatorname{Prim}(M(A)) \backslash \tilde{U}(e)$, recall that we write $Q \sim_{e} R$ if there is a net $\left(P_{\alpha}\right)$ in $\operatorname{Prim}(A) \backslash U(e)$ such that $\tilde{P}_{\alpha} \rightarrow Q, R$.

Proposition 5.2. Let $A$ be a $\sigma$-unital $C_{0}(X)$-algebra with base map $\phi$ and let $E$ be a nonempty closed subset of $X_{\phi}$. Consider the following conditions

(i) $H_{E}$ is strictly closed.

(ii) $J_{E}$ is locally modular and $\phi$ is locally closed at $E$.

Then (ii) $\Rightarrow(\mathrm{i})$, and (i) and (ii) are equivalent if $A$ is separable.

Proof. With the notation above, we have $J_{e}=J_{E}$ and $H_{e}=H_{E}$ by Proposition 2.9. Hence (ii) $\Rightarrow$ (i) follows immediately from Proposition 3.4.

Conversely, suppose that $A$ is separable and that (i) holds. Then $H_{e}$ is strictly closed by Proposition 2.9 and it follows from Corollary 3.10 that $J_{e}=J_{E}$ is locally modular and $q \circ \phi$ is locally closed at $e$. Hence $\phi$ is locally closed at $E$ by definition.

We are now ready for the main theorem of the section.

Theorem 5.3. Let $A$ be a $\sigma$-unital $C_{0}(X)$-algebra and suppose that $\phi$ is the complete regularization map for $\operatorname{Prim}(A)$ and that $\operatorname{Orc}(A)<\infty$. Let $E$ be a non-empty closed subset of $X_{\phi}$ and suppose that $J_{E}$ is locally modular. Then $\phi$ is locally closed at $E$ and $H_{E}$ is strictly closed.

Proof. As before, let $q: X_{\phi} \rightarrow X_{\phi} / E$ be the quotient map and $\{e\}=q(E)$. By Proposition 5.2, it suffices to show that $\phi$ is locally closed at $E$. Let $V$ be a proper open subset of $\operatorname{Prim}(A)$ with $V \supseteq H(e)$. It suffices to produce a continuous function $g$ on $\operatorname{Prim}(A)$ with $g(\operatorname{Prim}(A) \backslash V)=\{1\}$ and $g(H(e))=\{0\}$ (for then $g$ induces a continuous function on $\phi(\operatorname{Prim}(A))$ separating $\phi(\operatorname{Prim}(A) \backslash V)$ and $E$, so that $\overline{\phi(\operatorname{Prim}(A) \backslash V)} \cap E=\emptyset)$.

Set $Y=\left\{Q \in \operatorname{Prim}(A) \backslash U(e): \exists R \in \operatorname{Prim}(M(A) / A)\right.$ with $\left.\tilde{Q} \sim_{e} R\right\}$. Then $Y \cap H(e)$ is empty by Lemma 3.3. We claim that $Y$ is a closed subset of $\operatorname{Prim}(A)$. To see this, let 
$\left(Q_{\alpha}\right)$ be a net in $Y$ with $Q_{\alpha} \rightarrow Q \in \operatorname{Prim}(A)$. Then $Q \notin U(e)$ since $\operatorname{Prim}(A) \backslash U(e)$ is closed. By definition of $Y$, there is a net $\left(R_{\alpha}\right)$ in $\operatorname{Prim}(M(A) / A)$ with $\tilde{Q}_{\alpha} \sim_{e} R_{\alpha}$ for each $\alpha$. By the compactness of $\operatorname{Prim}(M(A) / A)$, there is a subnet $\left(R_{\beta}\right)$ of $\left(R_{\alpha}\right)$ with $R_{\beta} \rightarrow R \in$ $\operatorname{Prim}(M(A) / A)$. Then $\tilde{Q} \sim_{e} R$ and so $Q \in Y$, as required.

Next we note that $Y^{1}$ is closed in $\operatorname{Prim}(A)$. To see this, let $\left(Q_{\alpha}\right)$ be a net in $Y^{1}$ with $Q_{\alpha} \rightarrow Q \in \operatorname{Prim}(A)$. Then $Q \notin U(e)$, for otherwise eventually $Q_{\alpha} \in U(e) \subseteq H(e)$, which is impossible since $H(e)$ is a $\sim$-saturated set disjoint from $Y$. Let $\left(P_{\alpha}\right)$ be a net in $Y$ such that $P_{\alpha} \sim Q_{\alpha}$ for each $\alpha$. Since $P_{\alpha}, Q_{\alpha} \notin H(e), \tilde{P}_{\alpha} \sim_{e} \tilde{Q}_{\alpha}$. By the compactness of $\operatorname{Prim}(M(A))$ there exists $R \in \operatorname{Prim}(M(A))$ and a subnet $\left(P_{\beta}\right)$ of $\left(P_{\alpha}\right)$ such that $\tilde{P}_{\beta} \rightarrow R$. Hence $R \sim_{e} \tilde{Q}$. If $R=\tilde{S}$ for some $S \in Y$ then $Q \in Y^{1}$ as required. Otherwise $R \in \operatorname{Prim}(M(A) / A)$ and hence $Q \in Y \subseteq Y^{1}$. Thus $Y^{1}$ is closed.

Since $A$ is $\sigma$-unital, $\operatorname{Prim}(A)$ is $\sigma$-compact and hence every closed subset of $\operatorname{Prim}(A)$ is a Lindelof space. Thus, since $H(e)$ is closed and $\sim$-saturated, we may apply Lemma 5.1 to $H(e)$ and $Y$ to obtain disjoint open subsets $V^{\prime}$ and $V^{\prime \prime}$ of $\operatorname{Prim}(A)$ with $H(e) \subseteq V^{\prime}$ and $Y \subseteq V^{\prime \prime}$. By intersecting $V^{\prime}$ with the open set $V$ of the first paragraph, we may assume that $V^{\prime} \subseteq V$. Set $Z=\operatorname{Prim}(A) \backslash V^{\prime}$. Then $Z \supseteq V^{\prime \prime} \supseteq Y$ and the boundary of $Z$ in $\operatorname{Prim}(A)$ does not meet $V^{\prime \prime}$. Let $k=\operatorname{Orc}(A)$. Then the same argument that showed that $Y^{1}$ is closed also shows, inductively, that $Z^{1}, \ldots, Z^{k}$ are closed. Note that if $Q$ belongs to the boundary of $Z^{k}$ in $\operatorname{Prim}(A)$ then $Q \notin V^{\prime \prime}$ and so $Q \notin Y$. Thus there does not exist $R \in \operatorname{Prim}(M(A) / A)$ with $\tilde{Q} \sim_{e} R$.

Note that the disjoint sets $Z^{k}$ and $H(e)$ are $\sim$-saturated and hence so is the set $F:=$ $\operatorname{Prim}(A) \backslash\left(Z^{k} \cup H(e)\right)$. We now define an equivalence relation $\diamond$ on $\operatorname{Prim}(\mathrm{A})$ as follows. The $\diamond$-equivalence classes are: $Z^{k}, H(e)$, and the $\sim$-components of $F$. Set $W=\operatorname{Prim}(A) / \diamond$, equipped with the quotient topology, and let $p: \operatorname{Prim}(A) \rightarrow W$ be the quotient map. We show that $p$ is a closed map. Let $T$ be a closed subset of $\operatorname{Prim}(A)$ and set $T^{\prime}=p^{-1}(p(T))$. Let $\left(Q_{\alpha}\right)$ be a net in $T^{\prime}$ with a limit $Q \in \operatorname{Prim}(A)$. We must show that $Q \in T^{\prime}$. For each $Q_{\alpha}$ there exists $R_{\alpha} \in T$ such that $Q_{\alpha} \diamond R_{\alpha}$, and by the compactness of $\operatorname{Prim}(M(A))$ and by passing to subnets of $\left(Q_{\alpha}\right)$ and $\left(R_{\alpha}\right)$, if necessary, we may assume that there exists $R \in \operatorname{Prim}(M(A))$ with $\tilde{R}_{\alpha} \rightarrow R$.

We consider various cases. If $\left(Q_{\alpha}\right)$ is frequently in $Z^{k}$ then $Q \in Z^{k}$, since $Z^{k}$ is closed. Hence $Q \diamond Q_{\alpha}$ for $Q_{\alpha} \in Z^{k}$ and so $Q \in T^{\prime}$ since $T^{\prime}$ is $\diamond$-saturated. A similar argument shows that $Q \in T^{\prime}$ if $\left(Q_{\alpha}\right)$ is frequently in $H(e)$. Hence we may restrict attention to the case when $Q_{\alpha} \in F$ for all $\alpha$. This implies that $d_{A}\left(Q_{\alpha}, R_{\alpha}\right) \leq k$ and hence that for each $\alpha$ there is a walk $Q_{\alpha} \sim Q_{\alpha}^{1} \sim \ldots \sim Q_{\alpha}^{k}=R_{\alpha}$ (possibly with repetitions) of length $k$ between $Q_{\alpha}$ and $R_{\alpha}$. Hence $\tilde{Q}_{\alpha} \sim_{e} \tilde{Q}_{\alpha}^{1} \sim_{e} \ldots \sim_{e} \tilde{Q}_{\alpha}^{k}=\tilde{R}_{\alpha}$. Using the compactness of $\operatorname{Prim}(M(A)) \backslash \tilde{U}(e)$, and passing to successive subnets, we obtain a walk $\tilde{Q} \sim_{e} Q^{1} \sim_{e} \ldots \sim_{e} Q^{k}=R$ of length $k$ in $\operatorname{Prim}(M(A)) \backslash \tilde{U}(e)$ such that $\tilde{Q}, Q^{1}, \ldots, Q^{k}$ all lie in $(\tilde{F})^{-}$, the closure of $\tilde{F}$ in $\operatorname{Prim}(M(A))$.

Suppose that $P \in F^{-}$(the closure of $F$ in $\left.\operatorname{Prim}(A)\right)$ and that $\tilde{P} \sim_{e} P^{\prime}$ for some $P^{\prime} \in(\tilde{F})^{-}$. Then $P \notin U(e)$ and $P \notin Y$ and hence, by the definition of $Y, P^{\prime}=\tilde{S}$ for some $S \in \operatorname{Prim}(A)$. Furthermore, $S \in F^{-}$because $\tilde{S} \in(\tilde{F})^{-}$. Since $Q \in F^{-}$, it follows by induction that $Q^{i}=\widetilde{S}_{i}$ $(1 \leq i \leq k)$ for some $S_{i} \in \operatorname{Prim}(A)$, and hence that $Q \diamond S_{k}$. But $S_{k} \in T$, since $T$ is closed in $\operatorname{Prim}(A)$, and hence $Q \in T^{\prime}$ as required. Thus we have shown that $p$ is a closed map.

Now let $C$ and $D$ be non-empty, disjoint closed subsets of $W$. Then $C^{\prime}:=p^{-1}(C)$ and $D^{\prime}:=p^{-1}(D)$ are disjoint closed $\sim$-saturated subsets of $\operatorname{Prim}(A)$. Thus $C^{\prime}$ and $D^{\prime}$ are 
Lindelof and so Lemma 5.1 implies the existence of disjoint open sets $E^{\prime}$ and $F^{\prime}$ containing $C^{\prime}$ and $D^{\prime}$ respectively. We now use a standard characterization (see e.g. [24, §13.XIV Theorem 3]): a quotient map $r: M \rightarrow N$ is closed if and only if whenever $d \in N$ and $O$ is an open set containing $r^{-1}(d)$ then there exists a saturated open set $H$ such that $r^{-1}(d) \subseteq H \subseteq O$ (where $H$ is saturated if $\left.H=r^{-1}(r(H))\right)$. Applying this characterization in the present case to each of the points of $C$ and $D$ relative to $E^{\prime}$ and $F^{\prime}$ we obtain $\diamond$-saturated open sets $E^{\prime \prime}$ and $F^{\prime \prime}$ such that $C^{\prime} \subseteq E^{\prime \prime} \subseteq E^{\prime}$ and $D^{\prime} \subseteq F^{\prime \prime} \subseteq F^{\prime}$. Hence $p\left(E^{\prime \prime}\right)$ and $p\left(F^{\prime \prime}\right)$ are disjoint open sets of $W$ containing $C$ and $D$ respectively. Thus $W$ is normal.

It follows that there is a positive continuous function $f$ on $W$ with $\|f\|=1$ such that $f\left(p\left(Z^{k}\right)\right)=\{1\}$ and $f(p(H(e)))=\{0\}$. Then $g=f \circ p$ is a continuous function on $\operatorname{Prim}(A)$ with $g\left(Z^{k}\right)=\{1\}$ and $g(H(e))=\{0\}$. Since $\operatorname{Prim}(A) \backslash V \subseteq Z^{k}, g$ has the property required at the start of the proof.

Now let $A$ be a $C_{0}(X)$-algebra with base map $\phi$. Then $\phi$ factors as $\phi=\psi \circ \phi_{A}$ where $\phi_{A}$ is the complete regularization map and $\psi$ is continuous. The advantage of the following result over Proposition 5.2 is that $\psi$ is a map between completely regular spaces and should therefore be simpler to analyze.

Let $X_{\phi_{A}}$ denote the image of $\operatorname{Prim}(A)$ under the complete regularization map $\phi_{A}$. Then $\psi$ is a map from $X_{\phi_{A}} \rightarrow X_{\phi}$. By analogy with our earlier definition, we say that $\psi$ is locally closed at a non-empty subset $E \subseteq X_{\phi}$ if whenever $W$ is a closed subset of $X_{\phi_{A}}$ with $\psi(W) \cap E=\emptyset$ (i.e. $W \cap \psi^{-1}(E)=\emptyset$ ) then $\overline{\psi(W)} \cap E=\emptyset$.

Corollary 5.4. Let $A$ be a $\sigma$-unital $C_{0}(X)$-algebra with base map $\phi$ and suppose that $\operatorname{Orc}(A)<\infty$. Write $\phi=\psi \circ \phi_{A}$ where $\phi_{A}$ is the complete regularization map for $\operatorname{Prim}(A)$. Let $E$ be a non-empty closed subset of $X_{\phi}$. Consider the following conditions:

(i) $H_{E}$ is strictly closed;

(ii) $J_{E}$ is locally modular and $\psi: X_{\phi_{A}} \rightarrow X_{\phi}$ is locally closed at $E$. Then (ii) $\Rightarrow(\mathrm{i})$, and (i) and (ii) are equivalent if $A$ is separable.

Proof. Set $H(E)=\phi^{-1}(E)$. First, suppose that (ii) holds and let $Y$ be a closed subset of $\operatorname{Prim}(A)$ with $Y \cap H(E)$ empty. Let $W$ be the closure of $\phi_{A}(Y)$ in $X_{\phi_{A}}$. Then $W$ does not meet $\phi_{A}(H(E))=\psi^{-1}(E)$ by Theorem 5.3. Hence $\psi(W) \cap E=\emptyset$ and so the closure of $\psi(W)$ in $X_{\phi}$ does not meet $E$, since $\psi$ is locally closed at $E$. But $\phi(Y) \subseteq \psi(W)$ and hence $\overline{\phi(Y)} \cap E=\emptyset$. Thus $\phi$ is locally closed at $\mathrm{E}$ and so $J_{E}$ is strictly closed by Proposition 5.2.

Conversely, suppose that (i) holds and that $A$ is separable. Then $J_{E}$ is locally modular and $\phi$ is locally closed at $E$ by Proposition 5.2. Let $W$ be a closed set in $X_{\phi_{A}}$ such that $W \cap \psi^{-1}(E)$ is empty. Set $Y=\phi_{A}^{-1}(W)$. Then $Y$ is closed in $\operatorname{Prim}(A)$ and $Y \cap H(E)$ is empty. Hence the closure of $\phi(Y)$ does not meet $E$. But $\phi(Y)=\psi(W)$ and hence $\overline{\psi(W)} \cap E=\emptyset$. Thus $\psi$ is locally closed at $E$.

In particular, if $A$ in Corollary 5.4 is separable and $\psi$ is a closed map (for example, the identity map when $\phi=\phi_{A}$ ) then $H_{E}$ is strictly closed if and only if $J_{E}$ is locally modular.

\section{LOCALLY MODULAR IDEALS}

In this final section we look at locally modular ideals in the case when $\phi$ is the complete regularization map and $\operatorname{Orc}(A)<\infty$. We saw immediately after the definition of local modularity that there are two 'easy' ways for $J_{x}$ to be locally modular: if $x \in U_{\phi}$ or if $x$ is 
an isolated point in $X_{\phi}$. Example 4.8 gave two examples where $J_{x}$ is locally modular with $x \in \partial U_{\phi}$, and in the first of these $H(x)$ has empty interior in $\operatorname{Prim}(A)$. We will show that such behaviour cannot occur when $\phi$ is the complete regularization map and $\operatorname{Orc}(A)<\infty$. In this case, if $J_{x}$ is locally modular then either $x \in U_{\phi}$ or $H(x)$ has non-empty interior (Corollary 6.3).

Recall that for a $\mathrm{C}^{*}$-algebra $A$, we say that $P, Q \in \operatorname{Prim}(A)$ belong to the same Glimm class if $f(P)=f(Q)$ for all continuous, bounded, real-valued functions $f$ on $\operatorname{Prim}(A)$ (equivalently, $\phi_{A}(P)=\phi_{A}(Q)$, where $\phi_{A}$ is the complete regularization map on $\left.\operatorname{Prim}(A)\right)$. The algebra $A+Z(M(A))$ in the next result was introduced by Dixmier [11].

Lemma 6.1. Let $A$ be a $C^{*}$-algebra with $\operatorname{Orc}(A)<\infty$ and let $C=A+Z(M(A))$. Then $\operatorname{Orc}(C) \leq 2 \operatorname{Orc}(A)+2$.

Proof. First note that $A$ is an essential ideal in $C$, so that $\operatorname{Prim}(A)$ is (homeomorphic to) a dense open subset of $\operatorname{Prim}(C)$. Suppose that $Q_{1}, Q_{2}$ are distinct elements of $\operatorname{Prim}(C) \backslash$ $\operatorname{Prim}(A)$. Then $Q_{i}=M_{i}+A$ where $M_{i}$ is a maximal ideal of $Z(M(A))$ containing $A \cap Z$ $(i=1,2)$. It follows that $Z(M(A))$ separates $Q_{1}$ and $Q_{2}$. Thus each Glimm class in $\operatorname{Prim}(C)$ contains at most one element of $\operatorname{Prim}(C) \backslash \operatorname{Prim}(A)$. Hence if $P_{1} \sim P_{2} \sim \ldots \sim P_{n}$ is a path in $\operatorname{Prim}(C)$ then at most one element from $\operatorname{Prim}(C) \backslash \operatorname{Prim}(A)$ can occur among the $P_{i}$ 's. It follows that $d_{C}\left(P_{1}, P_{n}\right) \leq 2 \operatorname{Orc}(A)+2$ as required.

The next theorem is a general result giving a dichotomy for $\sim$-components in $\operatorname{Prim}(A)$ for any $C^{*}$-algebra $A$ for which $\operatorname{Orc}(A)<\infty$.

Theorem 6.2. Let $A$ be a $C_{0}(X)$-algebra and suppose that $\phi$ is the complete regularization map for $\operatorname{Prim}(A)$ and that $\operatorname{Orc}(A)<\infty$. Let $T$ be $a \sim$-component of $\operatorname{Prim}(A)$, so that $T \subseteq H(x)$ for some $x \in X_{\phi}$. Then either

(i) $J_{x} \nsupseteq Z(A)$ and $T=H(x)$; or

(ii) $J_{x} \supseteq Z(A)$ and there exist $P \in T$ and $R \in \operatorname{Prim}(M(A))$ with $R \supseteq A$ such that $\tilde{P} \sim R$.

Proof. Suppose that $P \in T$ and $R \in \operatorname{Prim}(M(A))$ with $R \supseteq A$ and $\tilde{P} \sim R$. Then $\bar{\phi}(R)=$ $\bar{\phi}(\tilde{P})=\phi(P)=x$ and it follows from Lemma 2.5 that $J_{x} \supseteq Z(A)$. We must show, therefore, that if there do not exist $P \in T$ and $R \in \operatorname{Prim}(M(A))$ with $R \supseteq A$ such that $\tilde{P} \sim R$ then alternative (i) holds.

Suppose, then, that for all $P \in T$ and $R \in \operatorname{Prim}(M(A))$ with $R \supseteq A, \tilde{P} \not R$. Set $k=\operatorname{Orc}(A)$, and let $Q \in T$. Note that $\tilde{T}=\left\{R \in \operatorname{Prim}(M(A)): d_{M(A)}(\tilde{Q}, R) \leq k\right\}$, by the supposition that for all $P \in T$ and $R \in \operatorname{Prim}(M(A))$ with $R \supseteq A, \tilde{P} \nsim R$. Hence $\tilde{T}$ is a closed (and thus compact) subset of $\operatorname{Prim}(M(A))$ by [33, Corollary 2.3] applied $k$ times. Set $L=\operatorname{ker} \tilde{T}$, a closed ideal of $M(A)$. If $A+L$ were a proper ideal of $M(A)$ there would exist $R \in \operatorname{Prim}(M(A))$ such that $R \supseteq A+L$. Hence $R \supseteq L$ and so $R \in \tilde{T}$ since $\tilde{T}$ is a closed subset of $\operatorname{Prim}(M(A))$, but also $R \supseteq A$. This is a contradiction, and hence $A+L=M(A)$.

Now set $C=A+Z(M(A))$, and for each $P \in \operatorname{Prim}(A)$ let $P^{\prime}$ be the unique primitive ideal in $C$ such that $P^{\prime} \cap A=P$.

Let $T^{\prime}:=\left\{P^{\prime}: P \in T\right\}$. We claim that $K \nsim P^{\prime}$ whenever $P \in T$ and $K \in \operatorname{Prim}(C)$ with $K \supseteq A$. Supposing otherwise, there exist $P \in T, K \in \operatorname{Prim}(C)$ with $K \supseteq A$, and a net $\left(P_{\alpha}\right)$ in $\operatorname{Prim}(A)$ such that $P_{\alpha}^{\prime} \rightarrow P^{\prime}$ and $P_{\alpha}^{\prime} \rightarrow K$. Since $K \supseteq A$ and $C \subseteq M(A)=A+L$, $K \nsupseteq L \cap C$. Hence there exists $c \in L \cap C$ such that $\|c+K\|=1$. By lower semi-continuity, $\left\|c+P_{\alpha}^{\prime}\right\| \geq 1 / 2$ eventually. Hence $\left\|c+\tilde{P}_{\alpha}\right\| \geq 1 / 2$ eventually (because both $\left\|c+P_{\alpha}^{\prime}\right\|$ and 
$\left\|c+\tilde{P}_{\alpha}\right\|$ are equal to $\left\|\bar{\pi}_{\alpha}(c)\right\|$ where $\pi_{\alpha}$ is an irreducible representation of $A$ with kernel $P_{\alpha}$ and $\bar{\pi}_{\alpha}$ is its unique ultra-weakly continuous extension to $\left.A^{* *}\right)$. By the compactness of the set $\{S \in \operatorname{Prim}(M(A)):\|c+S\| \geq 1 / 2\}$, we may assume, without loss of generality, that $\tilde{P}_{\alpha} \rightarrow R$ in $\operatorname{Prim}(M(A))$, where $\|c+R\| \geq 1 / 2$.

Since $P_{\alpha}^{\prime} \rightarrow P^{\prime}$ we have $P_{\alpha} \rightarrow P$ and $\tilde{P}_{\alpha} \rightarrow \tilde{P}$. Thus $\tilde{P} \sim R$. By the supposition of the second paragraph, it must be that $R=\tilde{S}$ for some $S \in \operatorname{Prim}(A)$. Hence $S \in T$. Thus we have $c \in L \subseteq \tilde{S}=R$, contradicting the fact that $\|c+R\| \geq 1 / 2$. It follows, then, that $K \nsim P^{\prime}$ whenever $P \in T$ and $K \in \operatorname{Prim}(C)$ with $K \supseteq A$, and hence that $T^{\prime}$ is a $\sim$-component in $\operatorname{Prim}(C)$.

By Lemma 6.1, $\operatorname{Orc}(C) \leq 2 \operatorname{Orc}(A)+2<\infty$, and since $\operatorname{Prim}(C)$ is compact it follows that $T^{\prime}$ is a Glimm class in $\operatorname{Prim}(C)$ [33, Corollary 2.7]. It follows at once that $T$ is a Glimm class in $\operatorname{Prim}(A)$. Thus $T=H(x)$. Now, let $P \in T$, and let $\phi_{C}: \operatorname{Prim}(C) \rightarrow Y$ be the complete regularization map for $\operatorname{Prim}(C)$, where $Y$ is the space of Glimm ideals of $C$ with the induced completely regular topology. Then the set $W=\phi_{C}(\operatorname{Prim}(C) \backslash \operatorname{Prim}(A))$ is compact and hence closed in $Y$, and does not contain the point $\phi_{C}(P)$. Thus there exists a continuous function $f$ on $Y$ taking the value 1 on $\phi_{C}\left(P^{\prime}\right)$ and 0 on $W$. Since $f$ vanishes on $W$, it follows that the central element $z$ of $C$ induced by $f$ actually belongs to $A$. But $z \notin P^{\prime}$, and $P^{\prime} \supseteq P \supseteq J_{x}$, and thus $z \notin J_{x}$. Hence $J_{x} \nsupseteq Z(A)$.

Corollary 6.3. Let $A$ be a $C_{0}(X)$-algebra and suppose that $\phi$ is the complete regularization map for $\operatorname{Prim}(A)$ and that $\operatorname{Orc}(A)<\infty$. Let $x \in X_{\phi}$ with $J_{x}$ locally modular. Then either $x \in U_{\phi}$ or $H(x)$ has non-empty interior.

Proof. Suppose that $x \notin U_{\phi}$ and that $H(x)$ has empty interior. Let $T$ be a $\sim$-component of $H(x)$. By Theorem 6.2 there exists $P \in T$ and $R \in \operatorname{Prim}(M(A) / A)$ such that $\tilde{P} \sim R$. Since $H(x)$ has empty interior, $P \in \partial H(x)$ and $\tilde{P} \sim_{x} R$. By Lemma 3.3, $J_{x}$ is not locally modular.

We conclude with two examples of $x \in \partial U_{\phi}$ with $J_{x}$ locally modular. The first has $\operatorname{Orc}(A)<\infty$ and $H(x)$ with non-empty interior. The second has $\operatorname{Orc}(A)=\infty$ and $H(x)$ with empty interior, showing that the condition $\operatorname{Orc}(A)<\infty$ in Corollary 6.3 is not redundant.

Example 6.4. (i) $A C_{0}(X)$-algebra with $z \in \partial U_{\phi}$ such that $J_{z}$ is locally modular, $\phi$ is locally closed at $z$, and $H(z)$ has non-empty interior.

As in Example 4.8(i), let $Y=\left\{(x, y) \in \mathbf{R}^{2}: y \geq 0\right\}$ be the upper half-plane, and let $L=\{(x, y) \in Y: y=0\}$ be the $x$-axis. Set $B=C_{0}(Y)$ and $C=C_{0}(L)$, and let $\pi: B \rightarrow C$ be the surjective $*$-homomorphism given by $\pi(b)=\left.b\right|_{L}(b \in B)$. Let $H$ be a separable, infinite-dimensional Hilbert space, $B(H)$ the algebra of bounded operators on $H$, and $K(H)$ the algebra of compact operators on $H$. Let $\rho: C \rightarrow B(H)$ be a ${ }^{*}$-monomorphism such that $\rho(C) \cap K(H)=\{0\}$. Set $D=\rho(C)+K(H)$, a $\mathrm{C}^{*}$-subalgebra of $B(H)$. Note that each element $d \in D$ can be uniquely expressed in the form $d=g+T$ where $g \in \rho(C)$ and $T \in K(H)$.

Set $A=\{(b, d) \in B \oplus D: \rho(\pi(b))=g\}$. Then $A$ is separable. For $(x, y) \in Y$, let $\theta_{x, y}$ be the character on $A$ given by $\theta_{x, y}(b, d)=b((x, y))$. Set $G=\{(b, d) \in A: \pi(b)=0, T=0\}$. Since any irreducible representation of $A$ extends to an irreducible representation of $B \oplus D$ (on a possibly larger Hilbert space), $\operatorname{Prim}(A)=\left\{\operatorname{ker} \theta_{x, y}:(x, y) \in Y\right\} \cup\{G\}$. Note that $G \subseteq \operatorname{ker} \theta_{x, 0}$ for all $x \in R$. It follows that a subset $W$ of $\operatorname{Prim}(A)$ is closed if and only if (i) 
$\left\{(x, y) \in Y: \operatorname{ker} \theta_{x, y} \in W\right\} \cap Y$ is closed in $Y$ (with the usual topology), and (ii) if $G \in W$ then $\operatorname{ker} \theta_{x, 0} \in W$ for all $x \in \mathbf{R}$. In particular $\{G\}$ is an open subset of $\operatorname{Prim}(A)$.

Set $X_{\phi}=Y / L$ and let $q: Y \rightarrow X_{\phi}$ be the quotient map. Set $X=\beta X_{\phi}$. Define $\phi: \operatorname{Prim}(A) \rightarrow X_{\phi} \subseteq X$ by $\phi\left(\theta_{x, y}\right)=q(x, y)((x, y) \in Y)$ and $\phi(G)=q(0,0)$. Then $\phi$ is the complete regularization map for $\operatorname{Prim}(A)$ and $\phi(G)$ is non-isolated in $X_{\phi}$. For $(x, y) \in Y \backslash L$, $J_{q(x, y)}=\operatorname{ker} \theta_{x, y}$ while $J_{q(0,0)}=G$. Each point of $Y$ has a compact neighbourhood in $Y$ and hence $J_{x}$ is locally modular for each $x \in X_{\phi}$, although $A / G$ is non-unital. Since $Y$ is normal, the map $\phi$ is easily seen to be relatively closed and hence $H_{x}$ is strictly closed for each $x \in X_{\phi}$ by Proposition 3.4. Taking $z=q(0,0)=q(G)$, however, we have that

$$
H(q(0,0))=\left\{\operatorname{ker} \theta_{x, 0}: x \in \mathbf{R}\right\} \cup\{G\}
$$

and this has non-empty interior $\{G\}$.

(ii) $A C_{0}(X)$-algebra with $x \in \partial U_{\phi}$ such that $J_{x}$ is locally modular, $\phi$ is locally closed at $x$, and $H(x)$ has empty interior.

Let $A$ be the $\mathrm{C}^{*}$-algebra defined as follows (see [33, Example 2.8]). Let $B$ be the $\mathrm{C}^{*}$-algebra consisting of all continuous functions from the interval $[0,1]$ into the $2 \times 2$ complex matrices. Let $B(1)$ be the $\mathrm{C}^{*}$-subalgebra of $B$ consisting of those functions $f \in B$ satisfying $f\left(2^{-n}\right)=$ $\operatorname{diag}\left(\lambda_{2 n-1}(f), \lambda_{2 n}(f)\right),(n \geq 1)$, and $f(0)=\operatorname{diag}(\lambda(f), \lambda(f))$, for some complex numbers $\lambda(f), \lambda_{n}(f)(n \geq 1)$. Let $A=\left\{f \in B(1): \lambda_{2 n}(f)=\lambda_{2 n+1}(f) \quad(1 \leq n<\infty)\right.$ and $\left.\lambda(f)=0\right\}$. Then $A$ is separable.

For $y \in(0,1] \backslash\left\{2^{-n}: n \geq 1\right\}$, set $P_{y}=\{f \in A: f(y)=(0)\}$. Then

$$
\operatorname{Prim}(A)=\left\{P_{y}: y \in(0,1] \backslash\left\{2^{-n}: n \geq 1\right\}\right\} \cup\left\{\operatorname{ker}\left(\lambda_{i}\right): i=1,3,5, \ldots\right\} .
$$

Set $X_{\phi}=(0,1] / *$ where for $r, s \in(0,1], r * s$ if $r=s$ or if $r, s \in\left\{2^{-n}: n \geq 1\right\}$ and let $q:(0,1] \rightarrow X_{\phi}$ denote the quotient map. Set $X=\beta X_{\phi}$ and $\infty=q(1 / 2)$. Define $\phi: \operatorname{Prim}(A) \rightarrow X$ by $\phi\left(P_{y}\right)=y\left(y \in(0,1] \backslash\left\{2^{-n}: n \geq 1\right\}\right)$ and $\phi\left(\lambda_{i}\right)=\infty(i=1,3,5, \ldots)$. Then $\phi$ is the complete regularization map for $\operatorname{Prim}(A)$.

If $x \in X_{\phi} \backslash\{\infty\}$ then $J_{x} \nsupseteq Z(A)=Z^{\prime}(A)$ and hence $x \in U_{\phi}$ and $J_{x}$ is locally modular. It is easy to see directly that $J_{\infty}$ is also locally modular. But $A / J_{\infty}$ is non-unital, since $\operatorname{Prim}\left(A / J_{\infty}\right)=\left\{\operatorname{ker}\left(\lambda_{i}\right): i=1,3,5, \ldots\right\}$ is non-compact, and hence $J_{\infty} \supseteq Z(A)$. Thus $U_{\phi}=X_{\phi} \backslash\{\infty\}$ and $W_{\phi}=\{\infty\}$. We show that $\phi$ is a relatively closed map. Let $Y$ be a closed subset of $\operatorname{Prim}(A)$ and set $Y^{\prime}=\phi^{-1}(\phi(Y))$. Then $Y^{\prime}=Y$ if $Y \cap\left\{\operatorname{ker}\left(\lambda_{i}\right): i=1,3,5, \ldots\right\}$ is empty, and $Y^{\prime}=Y \cup\left\{\operatorname{ker}\left(\lambda_{i}\right): i=1,3,5, \ldots\right\}$ otherwise. In either case $Y^{\prime}$ is closed, and hence $\phi$ is relatively closed. It follows, therefore, from Proposition 3.4 that $H_{x}$ is strictly closed for every $x \in X_{\phi}$.

\section{REFERENCES}

[1] C. A. Akemann and S. Eilers, Non-commutative end theory, Pacific J. Math. 185 (1998), 47-88.

[2] C. A. Akemann, G. K. Pedersen and J. Tomiyama, Multipliers of $C^{*}$-algebras, J. Funct. Anal. 13 (1973), 277-301.

[3] P. Ara and M. Mathieu, Sheaves of $C^{*}$-algebras, Math. Nachr. 283 (2010), 21-39.

[4] R. J. Archbold, Topologies for primal ideals, J. London Math. Soc., (2) 36 (1987), 524-542.

[5] R. J. Archbold and C. J. K. Batty, On factorial states of operator algebras, III, J. Operator Theory, 15 (1986), 53-81.

[6] R. J. Archbold and D. W. B. Somerset, Multiplier algebras of $C_{0}(X)$-algebras, Münster J. Math, 4 (2011), 73-100. 
[7] R. J. Archbold and D. W. B. Somerset, Ideals in the multiplier and corona algebras of a $C_{0}(X)$ algebra, J. London Math. Soc., (2) 85 (2012), 365-381.

[8] E. Blanchard, Tensor products of $C(X)$-algebras over $C(X)$, Astérique 232 (1995), 81-92.

[9] R. C. Busby, Double centralizers and extensions of $C^{*}$-algebras, Trans. Amer. Math. Soc. 132 (1968), 79-99.

[10] J. Dauns and K. H. Hofmann, Representations of Rings by Continuous Sections, Memoir 83, American Math. Soc., Providence, R.I., 1968.

[11] J. Dixmier, Ideal center of a $C^{*}$-algebra, Duke Math. J. 35 (1968), 375-382.

[12] J. Dixmier, $C^{*}$-algebras, North-Holland, Amsterdam, 1977.

[13] M. Dupré, The Classification and Structure of $C^{*}$-Bundles, Memoir 222, American Math. Soc., Providence, R.I., 1979.

[14] M. Dupré and R. M. Gillette, Banach Bundles, Banach Modules, and Automorphisms of $C^{*}$-Algebras, Pitman, Boston, 1983.

[15] S. Echterhoff and D. P. Williams, Locally inner actions on $C_{0}(X)$-algebras, J. Operator Theory 45 (2001), 131-160.

[16] G. A. Elliott, Derivations of matroid $C^{*}$-algebras, II, Ann. Math. (2) 100 (1974), 407-422.

[17] J. M. G. Fell, The structure of algebras of operator fields, Acta Mathematica, 106 (1961), 233-280.

[18] L. Gillman and M. Jerison, Rings of Continuous Functions, Van Nostrand, New Jersey, 1960.

[19] J. Glimm, A Stone-Weierstrass theorem for C*-algebras, Ann. of Math. 72 (1960), 216-244.

[20] I. Hirshberg, M. Rørdam, and W. Winter, $C_{0}(X)$-algebras, stability and strongly self-absorbing C*-algebras, Math. Ann. 339 (2007), 695-732.

[21] R. V. Kadison and J. R. Ringrose, Fundamentals of the theory of operator algebras, Vol. II, Academic Press, London, 1986.

[22] G. Kasparov, Equivariant KK-theory and the Novikov conjecture, Invent. Math. 91 (1988), 147-201.

[23] D. Kucerovsky and P. W. Ng, Nonregular ideals in the multiplier algebra of a stable $C^{*}$-algebra, Houston J. Math. 33 (2007), 1117-1130.

[24] K. Kuratowski, Topology, vol.1, Academic Press, New York, 1966.

[25] R-Y. Lee, On the C*-algebras of operator fields, Indiana Univ. Math. J. 25 (1976), 303-314.

[26] H. X. Lin, Ideals of multiplier algebras of simple AF-algebras, Proc. Amer. Math. Soc. 104 (1988), $239-244$.

[27] H. X. Lin, Simple $C^{*}$-algebras with continuous scales and simple corona algebras, Proc. Amer. Math. Soc. 112 (1991), 871-880.

[28] M. Nilsen, C*-bundles and $C_{0}(X)$-algebras, Indiana Univ. Math. J. 45 (1996), 463-477.

[29] G. K. Pedersen, $C^{*}$-algebras and their Automorphism Groups, Academic Press, London, 1979.

[30] G. K. Pedersen, $\mathrm{SAW}^{*}$-algebras and corona $\mathrm{C}^{*}$-algebras, contributions to non-commutative topology, J. Operator Theory, 15 (1986), 15-32.

[31] F. Perera, Ideal structure of multiplier algebras of simple $C^{*}$-algebras with real rank zero, Can. J. Math. 53 (2001), 592-630.

[32] M. Rørdam, Ideals in the multiplier algebra of a stable $C^{*}$-algebra, J. Operator Th. 25 (1991), 283-298.

[33] D. W. B. Somerset, The inner derivations and the primitive ideal space of a $\mathrm{C}^{*}$-algebra, J. Operator Theory, 29 (1993), 307-321.

[34] J. Tomiyama, Topological representations of $\mathrm{C}^{*}$-algebras, Tohoku Math. J. 15 (1963), 96-102.

[35] D. P. Williams, Crossed Products of $C^{*}$-algebras, American Mathematical Society, Rhode Island, 2007.

Institute of Mathematics, University of Aberdeen, King's College, Aberdeen AB24 3 Ue, Scotland, United Kingdom

E-mail address: r.archbold@abdn.ac.uk

Institute of Mathematics, University of Aberdeen, King's College, Aberdeen AB24 3UE, Scotland, United Kingdom

E-mail address: somerset@quidinish.fsnet.co.uk 